\title{
Metabolomic profiling of rare cell populations isolated by flow cytometry from tissues
}

Andrew W. DeVilbiss ${ }^{1}$, Zhiyu Zhao ${ }^{1}$, Misty S. Martin-Sandoval ${ }^{1}$, Jessalyn M. Ubellacker ${ }^{1}$, Alpaslan Tasdogan ${ }^{1}$, Michalis Agathocleous ${ }^{1}$, Thomas P. Mathews ${ }^{1,2^{*}}$, Sean J. Morrison ${ }^{1,2 *}$

${ }^{1}$ Children's Research Institute and Department of Pediatrics, University of Texas Southwestern Medical Center, Dallas, Texas 75390, USA

${ }^{2}$ Howard Hughes Medical Institute, University of Texas Southwestern Medical Center, Dallas, Texas 75390, USA

*Co-corresponding authors: Thomas.Mathews@UTSouthwestern.edu;

Sean.Morrison@UTSouthwestern.edu

Impact statement: We developed a method for metabolomic analysis of small numbers of flow cytometrically isolated cells from rare cell populations such as hematopoietic stem cells and circulating cancer cells. 
Little is known about the metabolic regulation of rare cell populations because most metabolites are hard to detect in small numbers of cells. We previously described a method for metabolomic profiling of flow cytometrically-isolated hematopoietic stem cells (HSCs) that detects approximately 60 metabolites in 10,000 cells (Agathocleous et al., 2017). Here we describe a new method involving hydrophilic liquid interaction chromatography (HILIC) and high-sensitivity orbitrap mass spectrometry that detected approximately 160 metabolites in 10,000 HSCs, including many more glycolytic and lipid intermediates. We improved chromatographic separation, increased mass resolution, minimized ion suppression, extracted with acetonitrile, and eliminated sample drying. Most metabolites did not significantly change during cell preparation and sorting. We used this method to profile HSCs and circulating melanoma cells. HSCs exhibited increased glycerophospholipid metabolites relative to unfractionated bone marrow cells and altered purine biosynthesis after methotrexate treatment in vivo. Circulating melanoma cells were depleted for purine intermediates relative to subcutaneous tumors, suggesting they decrease purine synthesis during metastasis. These methods facilitate the routine metabolomic analysis of rare cell populations from tissues.

Introduction

Metabolomics is typically performed using millions of cells, often using cultured cells, whole tissues, or tumor specimens (Jang et al., 2018). This measures average metabolite levels across the cells in a specimen but is blind to metabolic differences among cells in the same sample. As a result, we have limited insight into metabolic heterogeneity among cells within tissues or tumors (Kim and DeBerardinis, 2019, Muir et al., 2018). This limitation is particularly apparent when considering rare cells, such as stem cells or circulating cancer cells, that may be metabolically different from other cells. The difficulty of performing metabolomics on small numbers of these cells is compounded by the need to purify them from tissues, introducing 
additional technical challenges for metabolic analyses (Binek et al., 2019, Llufrio et al., 2018, Lau et al., 2020).

It is extremely time consuming and difficult to isolate a million cells from a rare cell population by flow cytometry. One study isolated over 1 million highly enriched CD34FIt3Lineage-Sca- $1^{+}$c-kit ${ }^{+}$hematopoietic stem cells (HSCs) by flow cytometry but had to pool bone marrow samples from 120 mice to do it, precluding the analysis of multiple replicates or routine application of this approach (Takubo et al., 2013). Metabolomics has also been performed on hundreds of thousands of flow cytometrically-isolated Lineage - Sca- $1^{+} \mathrm{c}-\mathrm{kit}^{+}(\mathrm{LSK})$ cells (Naka et al., 2015, Karigane et al., 2016), a larger and more heterogeneous population of hematopoietic stem and progenitor cells. Since only a small minority of these cells are HSCs, this strategy provides limited insight into metabolite levels in HSCs themselves. Others have characterized the phenotypes of mutant mice or metabolism in cultured hematopoietic stem and progenitor cells (Simsek et al., 2010, Ito et al., 2012, Ito et al., 2016, Ito et al., 2019, Wang et al., 2014, Ansó et al., 2017). However, it remains difficult to routinely compare metabolite levels between HSCs and other hematopoietic progenitors.

Metabolites have been profiled in single cells (Evers et al., 2019, Comi et al., 2017). However, these studies often use very large cells like Xenopus eggs (Onjiko et al., 2015) or Aplysia neurons (Nemes et al., 2012). Other single cell analyses have focused on small numbers of metabolites or specific subsets of metabolites (Luo and Li, 2017). Single cell metabolomics methods often involve mass spectrometry without chromatographic separation, making it more difficult to identify the detected species (Duncan et al., 2019, Ali et al., 2019).

We recently described a method for metabolomic analysis of highly purified, flow cytometrically isolated CD $150^{+}$CD48-LSK HSCs that detected approximately 60 metabolites in 10,000 cells (Agathocleous et al., 2017). Cells were kept cold during the entire purification process and sorted directly into $80 \%$ methanol to immediately quench enzymatic activity and extract metabolites. This method revealed that HSCs take up more ascorbate than other 
hematopoietic cells and depend upon ascorbate for epigenetic regulation and leukemia suppression, though coverage of many metabolic pathways was limited.

The challenge of performing metabolomic analysis in rare cells is not limited to stem cells as illustrated by a paucity of information about the metabolic state of circulating cancer cells. Many tumors spontaneously shed cancer cells into the blood (Micalizzi et al., 2017) but these cells are extremely rare, limiting the amount of material for analysis. We have developed methods for the flow cytometric isolation and characterization of circulating human melanoma cells from the blood of xenografted mice (Piskounova et al., 2015, Tasdogan et al., 2020). These cells undergo reversible metabolic changes during metastasis to survive oxidative stress, but these changes are just beginning to be characterized. Mass spectrometric analysis of single circulating cancer cells from the blood of patients revealed metabolites that differed among various kinds of cancer cells (Hiyama et al., 2015, Abouleila et al., 2019). Fluorescent probes have also been used to characterize metabolism in circulating cancer cells (Li et al., 2019).

Here we present a new method for the metabolomic analysis of rare stem cell and cancer cell populations isolated by flow cytometry. We have increased the number of metabolites we can detect in 10,000 HSCs to approximately 160 . We also applied this method to circulating cancer cells. Using these methods, the levels of most metabolites did not significantly change during cell preparation and sorting.

\section{RESULTS}

\section{Chromatography and mass spectrometry}

In order to significantly increase the numbers of metabolites we detected in small numbers of flow cytometrically isolated cells, we re-examined the chromatography and mass spectrometry approaches we used. A key limitation is discriminating the low levels of metabolites present in small numbers of cells from background signals. Background reflects contamination from various sources as well as the co-association of salts with organic 
compounds in mass spectrometers to generate organic salt clusters (matrix ions) that obscure the detection of metabolites. We reasoned we could improve the signal to noise ratio in low abundance samples and reduce interference by matrix ions by transitioning to a mass spectrometer with higher mass resolving power.

We chose a Q-Exactive HF-X hybrid quadrupole-orbitrap mass spectrometer (ThermoScientific) because it offers four advantages over the triple-quadrupole mass spectrometer used in our previous method (Agathocleous et al., 2017). First, whereas the triplequadrupole instrument acquires data for a predetermined number of metabolites, the orbitrap instrument captures spectra for the full mass range (80 - 1200 Daltons) with each scan, greatly increasing the number of metabolites detected. Second, orbitrap mass analyzers have higher mass resolving power and higher mass accuracy, increasing the ability to discriminate relevant analytes from background ions. Third, through untargeted acquisition of product ion spectra, orbitrap instruments enable the comparison of spectra from experimental samples with annotated spectrum libraries for high-confidence identification of metabolites. Finally, compared to other orbitrap models, the HF-X front end optics increase the number of ions that can pass into the mass spectrometer, boosting the signal from low abundance analytes.

We also wondered if a hydrophilic interaction liquid chromatography (HILIC) system would improve the separation of polar metabolites as compared to the reverse phase chromatography method in our original study (Agathocleous et al., 2017). To test this, we extracted metabolites from $5 \times 10^{6}$ mouse whole bone marrow (WBM) cells in $500 \mu$ of $80 \%$ methanol, dried the extracts in a vacuum concentrator, and reconstituted in water for reverse phase chromatography or $80 \%$ methanol for HILIC. Polar analytes eluted from the reverse phase column between 3 and 5 minutes, and from the HILIC column between 2 and 15 minutes, indicating that HILIC improved polar metabolite separation (data not shown).

When we ran high abundance samples either by reverse phase or HILIC we identified hundreds of metabolites by spectral database matching and manual peak review (data not 
shown). However, the improved metabolite separation and peak quality we observed with HILIC yielded more high confidence identifications of metabolites via spectral database matching alone (Figure 1A). HILIC also enabled detection of early-eluting lipid metabolites, which were not detected using reverse phase chromatography. Finally, HILIC eliminated the requirement for sample drying, which can alter the levels of certain metabolites and increases contamination (Lu et al., 2017). Given these advantages, we selected HILIC for further method development. We also fundamentally changed our approach to data analysis. To determine which metabolites were detected in low abundance samples we created a list of metabolites with known masses and chromatographic retention times from the analysis of high abundance samples. We first used unbiased metabolite identification software (Compound Discoverer) to compare experimentally observed mass spectra with annotated spectrum libraries to identify 571 metabolites. We confirmed the identities of each metabolite in the library by reviewing the MS2 spectra for each metabolite. We confirmed the retention times and mass spectra for over 450 metabolites in the library by running chemical standards. This library was used to determine how many metabolites were detected in low abundance samples by manually analyzing chromatographic peaks derived from extracts of 100,000 WBM cells. This resulted in a low abundance library containing 283 detectable metabolites that was used for manual metabolite quantitation in low abundance samples. This manual approach was more time consuming but more accurate than relying upon automated peak-calling algorithms, which often failed to accurately integrate LC-MS peaks from low-abundance samples.

\section{Reducing sources of contamination}

Background signals arose from the staining medium in which we suspended the cells, the flow cytometer sheath fluid, the solvent we used to extract metabolites from the sorted cells, and the drying and reconstitution of samples prior to liquid chromatography/mass spectrometry (LC-MS) (Agathocleous et al., 2017). While these very low levels of background would be 
negligible when analyzing high abundance samples, they did interfere with the ability to detect some metabolites in low abundance samples.

When performing reverse phase separation, metabolites were extracted using $80 \%$ methanol and then dried in a vacuum concentrator so they could be reconstituted in water for chromatography (Agathocleous et al., 2017). Transitioning to HILIC made it possible to directly inject organic solvents into the column, without drying and reconstituting in water. To test if contamination was reduced by not drying in a vacuum concentrator, we sorted droplets of sheath fluid with no cells in volumes equivalent to that required to sort 10,000 cells and processed the samples side-by-side in three ways. Some samples were dried in a standard vacuum concentrator, then reconstituted in $80 \%$ methanol and injected into the HILIC column. Some samples were dried in a new vacuum concentrator housed in a HEPA-filtered PCR hood to minimize contamination from the air, then reconstituted in $80 \%$ methanol and injected into the HILIC column. The remaining samples were sorted into $80 \%$ methanol and injected directly into the HILIC column without drying. The highest level of background contamination was in the samples dried in the standard vacuum concentrator (Figure 1B). The lowest background was in the samples injected into the column without drying, suggesting drying increased contamination.

To test whether we could detect more metabolites above background in low abundance samples if we did not dry and reconstitute, we sorted samples of 10,000 WBM cells, along with sheath fluid negative controls, and processed the samples side-by-side either with drying in a standard vacuum concentrator, with drying in a new vacuum concentrator in a HEPA-filtered PCR hood, or without drying. We detected 98, 123, and 130 metabolites significantly above sheath fluid background in the samples dried in a standard vacuum concentrator, dried in the HEPA-filtered vacuum concentrator, and undried, respectively (Figure 1C). We thus detected more metabolites above background in low abundance samples if we injected the sample directly into the HILIC column and incorporated this approach into the method. 


\section{Acetonitrile extraction}

Metabolites are most commonly extracted from cells using miscible aqueous-organic solvents, with the elimination of proteins, non-soluble components, and cellular debris by centrifugation. Different metabolites require different solvents for extraction (Rabinowitz and Kimball, 2007). To test different solvents, we extracted metabolites from 100,000 pipetted WBM cells using $80 \%$ methanol in water, 40:40:20 acetonitrile:methanol:water, or $80 \%$ acetonitrile in water. Using HILIC and orbitrap mass spectrometry we detected an average of 317 metabolites in samples extracted with $80 \%$ acetonitrile and 266 or 273 metabolites in samples extracted with 80\% methanol or 40:40:20 methanol:acetonitrile:water, respectively (Figure 1D). While we observed considerable overlap in the metabolites detected using each solvent, $80 \%$ acetonitrile yielded a number of metabolites that were not detected using the other solvents (Figure 1E). For these reasons, we selected $80 \%$ acetonitrile for further method development.

\section{Ion suppression and cell numbers}

Ion suppression of metabolite signals can occur as a result of the salt in the phosphate buffered saline (PBS) sheath fluid used for flow cytometric sorting: 1 to $3 \mathrm{nl}$ of sheath fluid is sorted along with each cell depending on whether a $70 \mu \mathrm{m}$ or $100 \mu \mathrm{m}$ nozzle is used. Flow cytometry sheath fluid must contain salt in order to electrostatically charge droplets for sorting; sorting more cells also sorts more salt. When using reverse phase chromatography, we reduced the magnitude of ion suppression by using $0.5 x$ PBS as sheath fluid and a $70 \mu \mathrm{m}$ nozzle in 4way purity sort mode to minimize droplet volume (Agathocleous et al., 2017). After changing to HILIC, we retested whether $0.5 x$ PBS or the $70 \mu \mathrm{m}$ nozzle affected the number of metabolites we detected. We sorted 10,000 WBM cells using $0.5 x$ PBS or $1 x$ PBS sheath fluid and either a $70 \mu \mathrm{m}$ or a $100 \mu \mathrm{m}$ nozzle. We found that sorting with the $70 \mu \mathrm{m}$ nozzle increased the number of metabolites we could detect above background as compared to the $100 \mu \mathrm{m}$ nozzle, regardless of sheath fluid PBS concentration (Figure 1F). We found no significant difference in 
the number of metabolites detected above background using $0.5 x$ versus $1.0 x$ PBS (Figure 1F).

These results suggest that metabolite signals were less sensitive to ion suppression using HILIC, in which salt eluted after metabolites of interest.

Next we tested if the number of metabolites we detected above background increased with increasing numbers of cells. We pipetted $10,000,20,000,30,000,50,000$ or 100,000 WBM cells (in equal volumes of HBSS buffer) directly into $80 \%$ acetonitrile and quantitated metabolites. The number of metabolites detected above sheath fluid background increased significantly with increasing numbers of cells, from $157 \pm 4$ metabolites in 10,000 cells to $222 \pm$ 9 metabolites in 100,000 cells (Figure 1G). In the same experiment, we detected an average of $155 \pm 2$ metabolites from 10,000 flow cytometrically sorted WBM cells (Figure 1G). We thus detected similar numbers of metabolites in flow cytometrically sorted and unsorted samples.

\section{Effect of flow cytometry on metabolite levels}

To determine if metabolic differences between cells are preserved during cell sorting using the methods describe above, we sorted or pipetted 10,000 HNT-34 AML cells or 10,000 DND-41 T-ALL cells into $80 \%$ acetonitrile. We detected 143 to 167 metabolites above background in each sample. Principal component analysis revealed differences between sorted and pipetted AML cells whereas differences among sorted and pipetted ALL cells were more subtle (Figure 1H). Irrespective of whether cells were sorted or pipetted, similar differences were observed between AML and ALL cells. Among sorted samples, 81 metabolites significantly differed between AML and ALL cells while among pipetted samples, 69 of the same metabolites differed (Figure 1I). Approximately $85 \%$ of the metabolites that significantly differed among sorted cells also significantly differed among pipetted cells and $75 \%$ of the significant differences among pipetted samples also significantly differed among sorted samples. Of the 12 metabolites that significantly changed in sorted but not pipetted cells, 10 trended in the same direction. Of the 23 metabolites that significantly changed in pipetted but not sorted samples, 20 
trended in the same direction in the sorted cells. Thus, the vast majority of metabolites exhibited similar differences among AML and ALL cells irrespective of whether the cells were sorted.

To more systematically assess the similarity of pipetted and sorted samples, we plotted Log2-transformed fold change values between AML and ALL cells for all metabolites above background in sorted versus pipetted samples (Figure 1J). The slope of the regression was near $1(y=0.96 x-0.06)$ and the correlation was strong for most metabolites (Spearman correlation coefficient, $r=0.81$ ). When we restricted the analysis to metabolites that significantly differed between sorted AML and ALL cells (fold change $>2$, FDR $<0.01$ ), the correlation was even stronger $(y=0.95 x-0.08 ; r=0.92 ;$ Figure $1 K)$. While the levels of some metabolites did change during sorting, most metabolites strongly correlated in sorted and unsorted samples.

\section{Effect of time on metabolite levels}

It typically took up to 2 hours to sort HSCs into acetonitrile starting from when the mice were killed. We wondered to what extent metabolite levels changed over time during cell isolation. To test this, we quickly flushed bone marrow from long bones and made single cell suspensions in HBSS that we kept on ice. We pipetted 10,000 cell aliquots of WBM cells into acetonitrile at $5,15,30,60,120,180$, and 240 minutes after killing the mice then performed metabolomic analysis on each sample. At all time points we detected 170 to 179 metabolites above sheath fluid background (Figure $1 \mathrm{~L}$ ). Relative to the samples collected at 5 minutes, only 2 metabolites significantly changed $(p<0.01, F C>2)$ in the samples collected at 15 minutes

(Figure 1M). The number of metabolites that significantly changed increased over time, but most of the changes occurred by 120 minutes. At 120 minutes, 19 metabolites significantly increased and 6 metabolites significantly decreased relative to the 5 minute samples (Figure 1M; Figure 1, supplementary table 1 shows the metabolites that changed over time). Therefore, some metabolites did change over time, but these represented less than $15 \%$ of detected metabolites. 
To more broadly assess the similarity of the samples over time, we plotted Log2transformed values for all detected metabolites in 5 minute versus 120 minute samples (Figure $1 N)$. The slope of the regression was near $1(y=0.97 x+0.62)$ and the correlation was strong, $r$ $=0.98$. We also plotted non-transformed values for all detected metabolites in 5 minute versus 120 minute samples, observing a similarly high correlation (Figure 10). Finally, to most clearly show the differences between 5 and 120 minute samples, we plotted only metabolites with signal intensity $<1 \times 10^{8}$ (Figure 1P). Again, the slope of the regression was near $1(y=0.95 x+$ $1.1 \times 10^{6}$ ) and the correlation was strong, $r=0.97$. Thus, metabolite intensity values strongly correlated among samples that incubated on ice for different periods of time.

\section{Metabolomic profiling of HSC/MPPs}

To assess the metabolomic profile of HSCs/MPPs we sorted 10,000 cell aliquots of CD48-Lineage ${ }^{-S c a} 1^{+} \mathrm{c}-\mathrm{kit}^{+}$cells and WBM cells. CD48-Lineage ${ }^{-}$Sca $1^{+} \mathrm{c}-\mathrm{kit}^{+}$cells represent $0.05 \%$ of WBM cells and are a very highly enriched for HSCs and MPPs (Oguro et al., 2013). The metabolomic profiles of HSCs and MPPs are extremely similar (Agathocleous et al., 2017). We detected $160 \pm 15$ metabolites above sheath fluid background in HSCs/MPPs and $147 \pm 15$ in WBM samples (Figure 2A). A total of 98 metabolites significantly differed in abundance between HSCs/MPPs and WBM cells (FDR $<0.01$, Figure 2 - supplementary table 1 ). Of these 98 metabolites, 51 differed by at least 2.5 fold (Figure 2B). Of the 16 metabolites that Agathocleous et al. (Agathocleous et al., 2017) found to significantly differ between HSCs/MPPs and WBM cells, 13 also significantly differed, in the same direction, using the new method (Figure 2 figure supplement 1). The other 3 metabolites either were not detected using the new method or could not be quantitated accurately due to extraction conditions. Thus, the new method detected most of the metabolic differences between HSC/MPPs and WBM cells observed by Agathocleous et al. (Agathocleous et al., 2017), while also detecting 82 additional differences. 
Pathway enrichment analysis found only one pathway that was significantly enriched (FDR<0.01): 10 of 36 metabolites in the murine KEGG "glycerophospholipid metabolism" pathway significantly differed in abundance between HSC/MPPs and WBM cells. The biological significance of this is not clear. HSCs were enriched for many components of the Kennedy (cytidine diphosphate-choline) pathway (Li and Vance, 2008, Kennedy and Weiss, 1956), including choline, choline phosphate, CDP-choline, ethanolamine phosphate, glycerophosphorylcholine, glycerophosphorylethanolamine, and many phosphatidylcholines (PC), phosphatidylethanolamines (PE), lysophosphatidylcholines (Lyso-PC), and lysophosphatidylethanolamines (Lyso-PE) (Figure 2B - figure supplement 2). Acetylcholine and several phosphatidylserine (PS) species were depleted in HSC/MPPs as compared to WBM (Figure 2B - figure supplement 2). These results raise the possibility that glycerophospholipid synthesis is activated in HSC/MPPs relative to WBM; however, additional studies will be required in the future to test this. The prominence of phospholipids among the differences between HSCs/MPPs and WBM cells illustrates the ability of the new method to detect differences not detected by prior methods.

To determine whether metabolic perturbations in HSCs in vivo can be detected by this method, we treated mice for 3 days with methotrexate. Methotrexate inhibits dihydrofolate reductase (DHFR) and AICAR transaminase (ATIC), steps in de novo purine biosynthesis (Baggott et al., 1986). Methotrexate treatment did not significantly affect bone marrow cellularity or the frequencies of HSCs, MPPs, or LSK cells in the bone marrow (Figure 3A-D).

Methotrexate treatment also did not significantly affect the reconstituting potential of WBM cells upon competitive transplantation into irradiated mice (Figure 3E). Metabolomic analysis of $10,000 \mathrm{HSC} / \mathrm{MPPs}$ from the bone marrow of methotrexate-treated and control mice revealed that the only pathway that was significantly enriched among the metabolites that differed was "purine metabolism". While methotrexate would also be expected to alter folate metabolism, folate species are very difficult to detect by metabolomics (Zheng et al., 2018, Chen et al., 2017) 
and are not detected by our method. Given that methotrexate inhibits ATIC, AICAR levels would be expected to increase after methotrexate treatment (Cronstein et al., 1993, Baggott et al., 1986, Allegra et al., 1985). Consistent with this, AICAR levels were 88 fold higher in HSCs/MPPs from methotrexate-treated as compared to control mice (Figure 3F). The method was thus capable of detecting expected metabolic perturbations in HSCs in vivo.

\section{Metabolomic profiling of circulating cancer cells}

To assess whether the method is broadly applicable, we tested if we could detect metabolic differences between circulating melanoma cells from the blood and the primary subcutaneous tumors from which they arose. We have shown previously that when efficiently metastasizing human melanomas are subcutaneously transplanted into NSG mice, they spontaneously metastasize, giving rise to rare circulating melanoma cells in the blood and metastatic tumors (Piskounova et al., 2015, Tasdogan et al., 2020). We subcutaneously transplanted M405 patient-derived melanoma cells into NSG mice. When the subcutaneous tumors reached $2.5 \mathrm{~cm}$ in diameter, we euthanized the mice and isolated 10,000 cell aliquots of melanoma cells by flow cytometry from mechanically dissociated subcutaneous tumors as well as from the blood of the same mice. We pooled blood from 6-10 mice per sample to isolate 10,000 circulating melanoma cells.

We detected 145 and 154 metabolites above sheath fluid background in the subcutaneous tumor and circulating melanoma cell samples, respectively (Figure 3G). Pathway enrichment analysis of all metabolites that significantly differed between subcutaneous tumor and circulating melanoma cells found one pathway that significantly (FDR < 0.01) differed purine metabolism. Several purine biosynthesis intermediates were depleted in circulating melanoma cells as compared to subcutaneous tumors, including IMP, XMP, GMP, and AMP (Figure 3H-K; Figure 3 - figure supplement 1). Given that circulating melanoma cells experience high levels of oxidative stress (Piskounova et al., 2015, Tasdogan et al., 2020), these data raise 
the possibility that metastasizing melanoma cells reduce purine biosynthesis, and perhaps other anabolic pathways, to preserve NADPH for oxidative stress resistance.

\section{Discussion}

The new method for metabolomic analysis of rare cells significantly increased metabolite numbers and pathway coverage relative to our prior method (Agathocleous et al., 2017) (Fig. 4A and 4B). We improved signal to noise ratio by using HILIC and an orbitrap mass spectrometer. We decreased contamination by eliminating sample drying and improved chromatographic performance by extracting metabolites with $80 \%$ acetonitrile. In principle, this method can be used to analyze any cell population isolated by flow cytometry, though in practice it is most useful when cell numbers are limited.

Cells can undergo metabolic changes upon removal from their in vivo environment (Lau et al., 2020). This is a particular problem when cells are enzymatically dissociated at $37^{\circ} \mathrm{C}$, when they exchange metabolites with the dissociation medium, or when cells are sorted into buffers that require additional processing steps before cell lysis and metabolite extraction (Lau et al., 2020, Binek et al., 2019, Llufrio et al., 2018). For this reason, we have avoided analyzing enzymatically dissociated cells. To avoid changes in metabolites during cell processing, we worked quickly and kept the cells cold from the time they left the animal until they were sorted into acetonitrile. Cellular metabolism is immediately quenched by sorting into cold acetonitrile. The levels of most metabolites strongly correlated in sorted and unsorted samples (Figure $1 \mathrm{H}$ K). Some metabolites exhibited changes in levels over time during cell processing (Figure 1M) but the levels of most metabolites strongly correlated in samples at 5 and 120 minutes after removal from the mouse (Figure $1 \mathrm{~N}-\mathrm{P}$ ). Overall, these control experiments suggest that most metabolite levels were not significantly changed by cell preparation and sorting.

Transitioning to HILIC provided several advantages. First, we were able to avoid sample drying, which significantly reduced contamination (Figure 1B-C). Second, we reduced ion 
suppression compared to our prior reverse phase method because salt eluted after the metabolites of interest on HILIC columns (Figure 1F). Third, HILIC improved the separation of polar metabolites, such as central carbon metabolites, while also enabling the detection of many lipid species. Nonetheless, some metabolites are better resolved and detected by reverse phase chromatography. Therefore, while HILIC provided a net advantage for our purposes, adapting this low cell number method to other chromatographies could improve the detection of certain classes of metabolites.

The extraction conditions described above are not suitable for the quantitation of some metabolites, including those that spontaneously oxidize (Lu et al., 2018). For example, ascorbate spontaneously oxidizes upon extraction from cells (Washko et al., 1992); therefore, in our prior study we added EDTA to the extraction solvent to prevent spontaneous oxidation (Agathocleous et al., 2017). In an effort to devise a general method in the current study, we did not add EDTA to the extraction solvent and therefore did not measure ascorbate levels accurately. Consistent with this, ascorbate was one of the 3 metabolites that differed between HSCs and WBM cells in our prior study (Agathocleous et al., 2017) that we did not detect as different in the current study (Figure 2 - figure supplement 1). The other two were spermidine and betaine, which were not detected using the new method.

We observed differences in the abundance of glycerophospholipids between HSCs/MPPs and WBM cells. Functional studies will be required to assess the biological significance of this difference. Few studies have examined lipid metabolism in HSCs (Xie et al., 2019, Ito et al., 2012, Ito et al., 2016, Lee et al., 2018, Pernes et al., 2019), partly because methods have not been readily available to quantitate lipid levels in HSCs. The ability of the new method to detect more than 60 lipids in 10,000 HSCs may facilitate future studies of lipid metabolism in stem cells. We also performed metabolomics on circulating melanoma cells from xenografted mice. Cancer cells must undergo metabolic changes to survive oxidative stress 
during metastasis (Piskounova et al., 2015, Tasdogan et al., 2020). Better understanding the metabolic changes could reveal new therapeutic vulnerabilities to block cancer progression.

\section{METHODS}

\section{Isolation of hematopoietic cells for metabolomics}

Bone marrow cells were collected quickly and maintained at 0 to $4^{\circ} \mathrm{C}$ throughout the staining and isolation procedure to minimize metabolic changes. Mice were euthanized by cervical dislocation. Bones were rapidly dissected and stored on ice in Hank's Buffered Salt Solution without $\mathrm{Mg}^{2+}$ and $\mathrm{Ca}^{2+}$ (HBSS, Corning). Muscle was stripped from the bones, then they were crushed in $2.5 \mathrm{ml}$ of HBSS using a pre-cooled mortar and pestle, on ice. Bone marrow cells were filtered through a $40 \mu \mathrm{m}$ strainer into a $50 \mathrm{ml}$ conical tube. The cells were then stained with fluorochrome-conjugated antibodies against B220 (FITC, Tonbo), Gr-1 (FITC, Tonbo), Ter119 (FITC, Tonbo), CD2 (FITC, Tonbo), CD3 (FITC, Tonbo), CD5 (FITC, BioLegend), CD8 (FITC, Tonbo), c-kit (APC-eFluor780, eBiosciences), Sca-1 (PerCP-Cy5.5, BioLegend), CD48 (APC, eBiosciences), and CD150 (PE, BioLegend) for 30 minutes on ice. Beginning 10 minutes before adding the antibodies, and continuing after the antibodies had been added, para-magnetic beads conjugated to anti-c-kit antibodies (Miltenyi) were added to the cells to facilitate pre-enrichment of $\mathrm{c}-\mathrm{kit}^{+}$cells in samples from which HSCs were sorted. To ensure that WBM cells were processed in the same way, these samples were enriched by positive selection of para-magnetic beads bound to anti-CD45 antibodies (Miltenyi). Positive selection was performed in the cold room at $4^{\circ} \mathrm{C}$ using a QuadroMACS manual separator (Miltenyi) and LS Columns (Miltenyi). Cells were eluted from columns in $2 \mathrm{ml}$ of HBSS, centrifuged for 5 minutes at $300 \times$ g, and resuspended in HBSS with 4',6-diamidino-2phenylindole (DAPI, $1 \mu \mathrm{g} / \mathrm{ml}$, Sigma) for flow cytometry. The gating strategy for the isolation of HSCs/MPPs is depicted in Figure 3 - figure supplement 2A. 


\section{Isolation of melanoma cells for metabolomics}

Mice were transplanted subcutaneously with human melanoma cells and the cells were allowed to spontaneously metastasize until the subcutaneous tumors reached $2.5 \mathrm{~cm}$. At this point, single cell suspensions were obtained by dissociating tumors mechanically with a scalpel on ice followed by gentle trituration. Cells were filtered through a $40 \mu \mathrm{m}$ strainer to generate a single cell suspension. Blood was collected from mice by cardiac puncture with a syringe pretreated with citrate-dextrose solution (Santa Cruz).

Subcutaneous tumor and blood specimens were first incubated on ice for 10 minutes with Ammonium-Chloride-Potassium (ACK) lysing buffer to eliminate red blood cells. The cells were washed with PBS and then stained with antibodies prior to flow cytometry. All antibody staining was performed for 20 minutes on ice, followed by washing with PBS and centrifuging at $200 \times \mathrm{g}$ for 5 minutes. Cells were stained with directly conjugated antibodies against mouse CD45 (APC, Tonbo Biosciences), mouse CD31 (APC, Biolegend), mouse Ter119 (APC, Tonbo Biosciences) and human HLA-A, B, C (G46-2.6-FITC, BD Biosciences). Human melanoma cells were isolated as cells that were positive for HLA and DsRed (melanoma cells were tagged with constitutive DsRed before subcutaneous transplantation), and negative for mouse endothelial (CD31) and hematopoietic markers (CD45 and Ter119). Cells were washed with PBS and resuspended in DAPI ( $1 \mu \mathrm{g} / \mathrm{ml}$, Sigma) to eliminate dead cells from sorts and analyses. The flow cytometry gating strategies for the isolation of primary tumor cells and circulating melanoma cells are depicted in Figure 3 - figure supplement 2B-C.

\section{Preparation of cultured cells for metabolomics}

HNT-34 AML cells and DND-41 T-ALL cells were cultured in RPMI medium with 10\% fetal bovine serum and $1 \%$ penicillin/streptomycin. Cells were maintained at a density of $5 \times 10^{5}$ cells $/ \mathrm{ml}$, and cultured at $37^{\circ} \mathrm{C}$ with $5 \% \mathrm{CO}_{2}$. Cells were washed once with PBS, and resuspended at a density of $1 \times 10^{6}$ cells per $\mathrm{ml}$ in ice cold PBS for flow cytometry. Pipetted cells 
were isolated by pipetting $10 \mu \mathrm{l}$ (containing 10,000 cells) into $40 \mu \mathrm{l} 100 \%$ acetonitrile.

\section{Flow cytometer preparation}

Flow cytometers were thoroughly cleaned before sorting low abundance samples to minimize background. All flow cytometry was performed using a FACSAria II or a FACSAria Fusion (BD Biosciences). The fluidics shutdown protocols were performed using $80 \%$ ethanol before each sort. A clean, metabolomics-dedicated FACSAria sheath tank was rinsed with ultrapure water several times to reduce contamination, before being filled with $4 \mathrm{I}$ of $0.5 \mathrm{x}$ phosphate buffered saline (PBS) made from tablets (Sigma) dissolved in ultrapure water. The metabolomics sheath tank was connected to the sorter using a dedicated $0.22 \mu \mathrm{m}$ filter. The fluidics startup protocol was performed using freshly made $0.5 \times$ PBS sheath fluid. The sorter was configured to use a $70 \mu \mathrm{m}$ nozzle but before the nozzle was inserted two cycles of clean flow cell protocols were performed with Windex. The sheath fluid was then run through the flow cytometer without a nozzle for 5 minutes to flush Windex and any remaining debris from the flow cell. At the same time, the $70 \mu \mathrm{m}$ nozzle was sonicated for 5 minutes to remove contamination and debris, and the cleanliness of the nozzle was confirmed by microscopy. The sheath fluid stream was turned off and the sort chamber was cleaned with a lint-free wipe and cotton swabs. The nozzle was then inserted and the stream was turned on. The sample line was cleaned again by running a $5 \mathrm{ml}$ sample tube of Windex for 5 minutes, followed by ultrapure water for 5 minutes. Four-way purity sort mode was used to minimize droplet size. The cell sample, the sorting chamber, and the collection tube adapter were all maintained at $4^{\circ} \mathrm{C}$ during sorting.

\section{Sorting cells for metabolomics}

The Eppendorf tubes into which cells were sorted were loaded with $40 \mu \mathrm{l}$ of $100 \%$ acetonitrile (Optima, Fisher Scientific) or methanol (Optima, Fisher Scientific) before sorting. We used a freshly opened bag of clean Eppendorf tubes (USA Scientific) and filtered pipette tips. 
The Eppendorf tubes were maintained at $-20^{\circ} \mathrm{C}$ until just prior to sorting. Cell samples were filtered through a $40 \mu \mathrm{m}$ strainer before sorting. The flow rate was minimized to reduce shear stress. Just before sorting, the Eppendorf tubes were opened using a clean microfuge tube opener (USA Scientific) to avoid contamination. After sorting, the tubes were sealed, vortexed and centrifuged briefly to collect all the liquid in the bottom of the tube, and placed on dry ice. Metabolites were extracted by vortexing again for 1 minute at high speed, followed by centrifugation at $17,000 \times \mathrm{g}$ for 15 minutes at $4^{\circ} \mathrm{C}$. The supernatant was transferred to autosampler vials with low volume inserts and analyzed immediately by LC-MS (see details below).

\section{Liquid chromatography and mass spectrometry}

Liquid chromatography was performed with a Vanquish Flex UHPLC (Thermo Scientific). The reverse phase method used a Waters HSS C18 column $(2.1 \times 150 \mathrm{~mm}, 1.7 \mu \mathrm{m})$ with a binary solvent gradient. Mobile phase A was water with $0.1 \%$ formic acid and mobile phase $\mathrm{B}$ was acetonitrile with $0.1 \%$ formic acid. Gradient separation proceeded as follows: from 0 to 5 minutes, $0 \%$ B; from 5 minutes to 45 minutes mobile phase $B$ was ramped linearly from $0 \%$ to $100 \%$; from 45 minutes to 52 minutes, mobile phase B was held at $100 \%$; from 52 to 52.1 minutes, mobile phase B was ramped linearly to $0 \%$; from 52.1 to 60 minutes, mobile phase B was held at $0 \%$. Throughout the course of the method, the solvent flow rate was kept to 100 $\mu \mathrm{l} / \mathrm{minute}$ and column temperature was held at $30^{\circ} \mathrm{C}$.

The HILIC method used a Millipore Sigma ZIC-pHILIC column $(2.1$ x 150, $5 \mu \mathrm{m})$ with a binary solvent gradient. Mobile phase A was water containing $10 \mathrm{mM}$ ammonium acetate, $\mathrm{pH}$ 9.8 with ammonium hydroxide; mobile phase B was $100 \%$ acetonitrile. Gradient separation proceeded as follows: from 0 to 15 minutes mobile phase $B$ was ramped linearly from $90 \%$ to $30 \%$; from 15 minutes to 18 minutes, mobile phase B was held at 30\%; from 18 minutes to 19 minutes, mobile phase B was ramped linearly from $30 \%$ to $90 \%$; mobile phase B was held at $90 \%$ from 19 minutes to 27 minutes to regenerate the initial chromatographic environment. 
Throughout the method, solvent flow rate was kept at $250 \mu \mathrm{l} /$ minute and the column temperature was maintained at $25^{\circ} \mathrm{C}$. For low abundance samples, $20 \mu \mathrm{l}$ of sample was injected onto the column. For high abundance samples, $10 \mu \mathrm{l}$ was injected.

All mass spectrometry data were acquired using a Thermo Scientific (Bremen, Germany) QExactive HF-X mass spectrometer (LC-MS/MS). For low abundance samples, a polarityswitching MS1 only acquisition method was used. Each polarity was acquired at a resolving power of 120,000 full width at half maximum (FWHM); the automatic gain control (AGC) target was set to $1,000,000$ with a maximum inject time of 50 milliseconds. The scan range was set to 80-1200 Daltons. High-abundance samples analyzed for library construction were acquired with two separate ddMS2 methods - one for positive mode and another for negative mode.

Precursor MS1 data for this method were acquired with the exact same settings as those described above. Product ion MS data were acquired with a resolving power of 15,000 FWHM; the AGC target was set to 200,000 , with a maximum inject time of $150 \mathrm{~ms}$. A top-10 data dependent MS scheme was used with an isolation window of $1 \mathrm{Da}$ and an isolation offset of 0.5 Da. Analytes were fragmented with stepped collision energies of 30, 50 and 70 Normalized Collision Energy (NCE) units. The minimum AGC target was set to 8,000 with a dynamic exclusion of 30 seconds.

\section{Metabolite library development}

To develop the metabolite library we used to analyze samples, we acquired LC-MS/MS data from high abundance samples using a data dependent MS/MS method. Metabolites were identified in an unbiased fashion using Compound Discoverer 3.0 (ThermoScientific).

Metabolites were added to the initial library only if they met the following criteria. First, chromatographic peaks had to align in all samples, and peak intensity had to increase with cell number. Second, precursor mass accuracy had to be within 5 ppm of theoretical mass, with an naturally occurring isotope pattern that matched that predicted by the chemical formula. Third, 
the MS/MS product ion spectra had to either match an annotated database (mzCloud, Human Metabolome Data Base, Lipid Maps, and ChemSpider) or had to be confirmed by analysis of chemical standards. This process yielded a 590 metabolite library with known masses and chromatographic retention times. This library was imported into the manual peak review software Trace Finder 4.1 (ThermoScientific) for manual peak integration of all low abundance LC-MS data. To narrow this list of 590 metabolites to the metabolites that might be detected in 10,000 sorted cells, we determined which of the 590 metabolites were observed in 100,000 WBM cells. We found 289 metabolites that were detected in 100,000 WBM cells. This 289 metabolite library was used for manual analyses of LC-MS data from low abundance samples. When additional metabolites were observed in new experiments they were added to the library.

\section{Melanoma specimens}

Melanoma specimens were obtained with informed consent from all patients according to protocols approved by the Institutional Review Board (IRB) of the University of Michigan Medical School (IRBMED approvals HUM00050754 and HUM00050085 (Quintana et al., 2012)) and the University of Texas Southwestern Medical Center (IRB approval 102010-051). Materials used in the manuscript are available, though there are restrictions imposed by IRB requirements and institutional policy on the sharing of materials from patients.

\section{Mouse studies and xenograft assays}

All mouse experiments complied with all relevant ethical regulations and were performed according to protocols approved by the Institutional Animal Care and Use Committee at the University of Texas Southwestern Medical Center (protocols 2016-101360 and 2019-102632). For all experiments, mice were kept on normal chow and fed ad libitum. The mice used in all experiments were 8 to 12 week-old C57BL/Ka mice, with the exception of melanoma studies, which were subcutaneously xenografted into 4 to 8 week-old NOD.CB17-Prkdc $c^{\text {scid }} / / 2 r g^{\text {tm1 } 1 W_{j l} / S z J}$ 
(NSG) mice. Both male and female mice were used. For melanoma experiments, the maximum permitted tumor diameter was $2.5 \mathrm{~cm}$. Subcutaneous tumor diameters were measured weekly with calipers until any tumor in the mouse cohort reached $2.5 \mathrm{~cm}$ in its largest diameter. At that point, all mice in the cohort were killed, per approved protocol, for analysis of subcutaneous tumors and circulating melanoma cells. For each replicate, subcutaneous tumors and circulating melanoma cells were pooled from 6-10 mice.

\section{Methotrexate treatment}

8-12 week old C57BL/Ka mice were intraperitoneally injected daily with methotrexate (1.25 mg/kg/day) or DMSO vehicle control, for 3 days. Mice were sacrificed by cervical dislocation 2 hours after the final methotrexate dose and bone marrow cells were collected for analysis.

\section{Statistical analysis of metabolomic data}

We developed an R tool for the analysis of metabolite LC-MS peak intensity data. The data were visualized using multiple methods, including violin-box plots, histograms, clustered heatmaps, principle component analysis, and correlation plots to assess data quality and identify batch effects. To assess the statistical significance of differences in metabolite levels between samples we used R's Generalized Linear Models (GLM) (Dobson and Barnett, 2018) function with the Gaussian distribution on the log-transformed data. To compare metabolite levels in cell samples to sheath fluid samples we used GLM with $\log _{2}(x+1)$-transformed, nonnormalized data. Metabolites with fold change $>2$ and FDR $<0.05$ were considered above background. To assess the statistical significance of differences in metabolite levels between two types of cells, we normalized the cell samples using the Relative Log Expression (RLE) method (Anders and Huber, 2010), and $\log _{2}(x+1)$-transformed the normalized data. For all comparisons between samples, we used the half-minimum imputation to replace zero values 
with half of the minimum non-zero value for each metabolite, and used R's GLM method. To adjust for multiple comparisons we used the False Discovery Rate (FDR) method. When samples were from the same mice we used pairing as an independent variable in the GLM. When batch effects were observed we used batch as an independent variable in the GLM. R packages used by this tool include stats, openxlsx, data.table, gtools, matrixStats, cplm, ggplot2, cowplot, pheatmap, ggcorrplot, eulerr, and GGally.

The metabolomics data analysis tool can be downloaded from https://git.biohpc.swmed.edu/CRI/ODA for academic use. This tool includes an ODA.R script file, an accompanying Excel data template file, and example analyses. The script can be run from Linux/Maclntosh Terminal or Windows PowerShell using the Rscript command followed by the Excel input file name and the Excel output file name. R with the Rscript command (version 3.5.1 or later is recommended) and internet access are required to run this tool as other $\mathrm{R}$ packages must be auto-downloaded by the tool. Data should be entered into the Excel template and parameters for analysis selected. First-time users should read the instructions in the data template. The analysis reports and figures are saved together in the Excel output file. Figures are also saved in a folder in the .png and .ps formats. Example analysis results are provided to illustrate typical analysis settings and their outputs.

\section{Assessing statistical significance}

Mice were allocated to experiments randomly and samples processed in an arbitrary order, but formal randomization techniques were not used. Prior to analyzing the statistical significance of differences among treatments, we tested whether the data were normally distributed and whether variance was similar among treatments. To test for normal distribution, we performed the Shapiro-Wilk test when $3 \leq n<20$ or the D'Agostino Omnibus test when $n \geq 20$. To test if variability significantly differed among treatments, we performed $F$-tests (for 
experiments with two treatments) or Levene's median tests (for more than two treatments). When the data significantly deviated from normality or variability significantly differed among treatments, we log2-transformed the data and tested again for normality and variability. If the transformed data did not significantly deviate from normality and equal variability, we performed parametric tests on the transformed data. Fold change data were always log2-transformed.

All the statistical tests we used were two-sided, where applicable. To assess the statistical significance of a difference between two treatments, we used Student's t-tests or paired t-tests (when a parametric test was appropriate). To assess the statistical significance of differences between two cumulative frequency distributions, we used the Kolmogorov-Smirnov tests. Multiple Kolmogorov-Smirnov tests were followed by Holm-Sidak's multiple comparisons adjustment. To assess the statistical significance of differences between more than two treatments, we used repeated measures one-way or two-way ANOVAs (when a parametric test was appropriate) followed by Tukey's, Dunnet's, or Sidak's multiple comparisons adjustment. To assess the statistical significance of differences between transplant data, we used mixed-effects analysis (when a parametric test was appropriate and there were missing data points) followed by Sidak's multiple comparisons adjustment. To assess the correlation between two sets of samples, we calculated Spearman correlation coefficients $(r$, the data were not normally distributed) and performed linear regression analysis.

All statistical analyses were performed with Graphpad Prism 8.3. All data represent mean \pm standard deviation. Samples sizes were not pre-determined based on statistical power calculations but were based on our experience with these assays. No data were excluded; however, mice sometimes died during experiments, presumably due to complications associated with irradiation and bone marrow transplantation. In those instances, data that had already been collected on the mice in interim analyses were included (such as donor contribution to peripheral blood chimerism over time). 


\section{References}

Abouleila, Y., Onidani, K., Ali, A., Shoji, H., Kawai, T., Lim, C. T., Kumar, V., Okaya, S., Kato, K., Hiyama, E., Yanagida, T., Masujima, T., Shimizu, Y. \& Honda, K. 2019. Live single cell mass spectrometry reveals cancer-specific metabolic profiles of circulating tumor cells.

Cancer Sci, 110, 697-706.

Agathocleous, M., Meacham, C. E., Burgess, R. J., Piskounova, E., Zhao, Z., Crane, G. M., Cowin, B. L., Bruner, E., Murphy, M. M., Chen, W., Spangrude, G. J., Hu, Z., Deberardinis, R. J. \& Morrison, S. J. 2017. Ascorbate regulates haematopoietic stem cell function and leukaemogenesis. Nature, 549, 476-481.

Ali, A., Abouleila, Y., Shimizu, Y., Hiyama, E., Emara, S., Mashaghi, A. \& Hankemeier, T. 2019. Single-cell metabolomics by mass spectrometry: Advances, challenges, and future applications. TrAC Trends in Analytical Chemistry, 120, 115436.

Allegra, C. J., Drake, J. C., Jolivet, J. \& Chabner, B. A. 1985. Inhibition of phosphoribosylaminoimidazolecarboxamide transformylase by methotrexate and dihydrofolic acid polyglutamates. Proceedings of the National Academy of Sciences of the United States of America, 82, 4881-4885.

Anders, S. \& Huber, W. 2010. Differential expression analysis for sequence count data.

Genome Biology, 11, R106.

Ansó, E., Weinberg, S. E., Diebold, L. P., Thompson, B. J., Malinge, S., Schumacker, P. T., Liu, X., Zhang, Y., Shao, Z., Steadman, M., Marsh, K. M., Xu, J., Crispino, J. D. \& Chandel, N. S. 2017. The mitochondrial respiratory chain is essential for haematopoietic stem cell function. Nature cell biology, 19, 614-625.

Baggott, J. E., Vaughn, W. H. \& Hudson, B. B. 1986. Inhibition of 5-aminoimidazole-4carboxamide ribotide transformylase, adenosine deaminase and 5'-adenylate deaminase by 
polyglutamates of methotrexate and oxidized folates and by 5-aminoimidazole-4carboxamide riboside and ribotide. Biochem J, 236, 193-200.

Binek, A., Rojo, D., Godzien, J., Rupérez, F. J., Nuñez, V., Jorge, I., Ricote, M., Vázquez, J. \& Barbas, C. 2019. Flow Cytometry Has a Significant Impact on the Cellular Metabolome. J Proteome Res, 18, 169-181.

Chen, L., Ducker, G. S., Lu, W., Teng, X. \& Rabinowitz, J. D. 2017. An LC-MS chemical derivatization method for the measurement of five different one-carbon states of cellular tetrahydrofolate. Anal Bioanal Chem, 409, 5955-5964.

Comi, T. J., Do, T. D., Rubakhin, S. S. \& Sweedler, J. V. 2017. Categorizing Cells on the Basis of their Chemical Profiles: Progress in Single-Cell Mass Spectrometry. Journal of the American Chemical Society, 139, 3920-3929.

Cronstein, B. N., Naime, D. \& Ostad, E. 1993. The antiinflammatory mechanism of methotrexate. Increased adenosine release at inflamed sites diminishes leukocyte accumulation in an in vivo model of inflammation. The Journal of clinical investigation, 92, $2675-2682$.

Dobson, A. J. \& Barnett, A. G. 2018. An introduction to generalized linear models, CRC press. Duncan, K. D., Fyrestam, J. \& Lanekoff, I. 2019. Advances in mass spectrometry based singlecell metabolomics. Analyst, 144, 782-793.

Evers, T. M. J., Hochane, M., Tans, S. J., Heeren, R. M. A., Semrau, S., Nemes, P. \& Mashaghi, A. 2019. Deciphering Metabolic Heterogeneity by Single-Cell Analysis. Analytical Chemistry, 91, 13314-13323.

Hiyama, E., Ali, A., Amer, S., Harada, T., Shimamoto, K., Furushima, R., Abouleila, Y., Emara, S. \& Masujima, T. 2015. Direct Lipido-Metabolomics of Single Floating Cells for Analysis of Circulating Tumor Cells by Live Single-cell Mass Spectrometry. Analytical Sciences, 31, $1215-1217$. 
Ito, K., Carracedo, A., Weiss, D., Arai, F., Ala, U., Avigan, D. E., Schafer, Z. T., Evans, R. M., Suda, T., Lee, C. H. \& Pandolfi, P. P. 2012. A PML-PPAR-ס pathway for fatty acid oxidation regulates hematopoietic stem cell maintenance. Nat Med, 18, 1350-8.

Ito, K., Turcotte, R., Cui, J., Zimmerman, S. E., Pinho, S., Mizoguchi, T., Arai, F., Runnels, J. M., Alt, C., Teruya-Feldstein, J., Mar, J. C., Singh, R., Suda, T., Lin, C. P., Frenette, P. S. \& Ito, K. 2016. Self-renewal of a purified \&lt;em\&gt;Tie2\&lt;/em\&gt;\&lt;sup\&gt;+\&lt;/sup\&gt; hematopoietic stem cell population relies on mitochondrial clearance. Science, 354, 1156.

Ito, K., Bonora, M. \& Ito, K. 2019. Metabolism as master of hematopoietic stem cell fate. Int J Hematol, 109, 18-27.

Jang, C., Chen, L. \& Rabinowitz, J. D. 2018. Metabolomics and Isotope Tracing. Cell, 173, 822837.

Karigane, D., Kobayashi, H., Morikawa, T., Ootomo, Y., Sakai, M., Nagamatsu, G., Kubota, Y., Goda, N., Matsumoto, M., Nishimura, Emi k., Soga, T., Otsu, K., Suematsu, M., Okamoto, S., Suda, T. \& Takubo, K. 2016. p38a Activates Purine Metabolism to Initiate Hematopoietic Stem/Progenitor Cell Cycling in Response to Stress. Cell Stem Cell, 19, 192-204.

Kennedy, E. P. \& Weiss, S. B. 1956. The function of cytidine coenzymes in the biosynthesis of phospholipides. J Biol Chem, 222, 193-214.

Kim, J. \& Deberardinis, R. J. 2019. Mechanisms and Implications of Metabolic Heterogeneity in Cancer. Cell Metabolism, 30, 434-446.

Lau, A. N., Li, Z., Danai, L. V., Westermark, A. M., Darnell, A. M., Ferreira, R., Gocheva, V., Sivanand, S., Lien, E. C., Sapp, K. M., Mayers, J. R., Biffi, G., Chin, C. R., Davidson, S. M., Tuveson, D. A., Jacks, T., Matheson, N. J., Yilmaz, O. \& Vander Heiden, M. G. 2020. Dissecting cell type-specific metabolism in pancreatic ductal adenocarcinoma. eLife, 9, e56782.

Lee, M. K. S., Al-Sharea, A., Dragoljevic, D. \& Murphy, A. J. 2018. Hand of FATe: lipid metabolism in hematopoietic stem cells. Current Opinion in Lipidology, 29. 
Li, Z. \& Vance, D. E. 2008. Thematic Review Series: Glycerolipids. Phosphatidylcholine and choline homeostasis. Journal of Lipid Research, 49, 1187-1194.

Li, Z., Wang, Z., Tang, Y., Lu, X., Chen, J., Dong, Y., Wu, B., Wang, C., Yang, L., Guo, Z., Xue, M., Lu, S., Wei, W. \& Shi, Q. 2019. Liquid biopsy-based single-cell metabolic phenotyping of lung cancer patients for informative diagnostics. Nature Communications, 10, 3856.

Llufrio, E. M., Wang, L., Naser, F. J. \& Patti, G. J. 2018. Sorting cells alters their redox state and cellular metabolome. Redox Biol, 16, 381-387.

Lu, W., Su, X., Klein, M. S., Lewis, I. A., Fiehn, O. \& Rabinowitz, J. D. 2017. Metabolite Measurement: Pitfalls to Avoid and Practices to Follow. Annual review of biochemistry, 86, 277-304.

Lu, W., Wang, L., Chen, L., Hui, S. \& Rabinowitz, J. D. 2018. Extraction and Quantitation of Nicotinamide Adenine Dinucleotide Redox Cofactors. Antioxid Redox Signal, 28, 167-179. Luo, X. \& Li, L. 2017. Metabolomics of Small Numbers of Cells: Metabolomic Profiling of 100, 1000, and 10000 Human Breast Cancer Cells. Analytical Chemistry, 89, 11664-11671.

Micalizzi, D. S., Maheswaran, S. \& Haber, D. A. 2017. A conduit to metastasis: circulating tumor cell biology. Genes \& development, 31, 1827-1840.

Muir, A., Danai, L. V. \& Vander Heiden, M. G. 2018. Microenvironmental regulation of cancer cell metabolism: implications for experimental design and translational studies. Dis Model Mech, 11, dmm035758.

Naka, K., Jomen, Y., Ishihara, K., Kim, J., Ishimoto, T., Bae, E. J., Mohney, R. P., Stirdivant, S. M., Oshima, H., Oshima, M., Kim, D. W., Nakauchi, H., Takihara, Y., Kato, Y., Ooshima, A. \& Kim, S. J. 2015. Dipeptide species regulate p38MAPK-Smad3 signalling to maintain chronic myelogenous leukaemia stem cells. Nat Commun, 6, 8039.

Nemes, P., Knolhoff, A. M., Rubakhin, S. S. \& Sweedler, J. V. 2012. Single-cell metabolomics: changes in the metabolome of freshly isolated and cultured neurons. ACS Chem Neurosci, $3,782-792$. 
Oguro, H., Ding, L. \& Morrison, S. J. 2013. SLAM family markers resolve functionally distinct subpopulations of hematopoietic stem cells and multipotent progenitors. Cell stem cell, 13, 102-116.

Onjiko, R. M., Moody, S. A. \& Nemes, P. 2015. Single-cell mass spectrometry reveals small molecules that affect cell fates in the 16-cell embryo. Proceedings of the National Academy of Sciences of the United States of America, 112, 6545-6550.

Pernes, G., Flynn, M. C., Lancaster, G. I. \& Murphy, A. J. 2019. Fat for fuel: lipid metabolism in haematopoiesis. Clin Transl Immunology, 8, e1098-e1098.

Piskounova, E., Agathocleous, M., Murphy, M. M., Hu, Z., Huddlestun, S. E., Zhao, Z., Leitch, A. M., Johnson, T. M., Deberardinis, R. J. \& Morrison, S. J. 2015. Oxidative stress inhibits distant metastasis by human melanoma cells. Nature, $527,186-91$.

Quintana, E., Piskounova, E., Shackleton, M., Weinberg, D., Eskiocak, U., Fullen, D. R., Johnson, T. M. \& Morrison, S. J. 2012. Human Melanoma Metastasis in NSG Mice Correlates with Clinical Outcome in Patients. Science Translational Medicine, 4, 159ra149.

Rabinowitz, J. D. \& Kimball, E. 2007. Acidic Acetonitrile for Cellular Metabolome Extraction from Escherichia coli. Analytical Chemistry, 79, 6167-6173.

Simsek, T., Kocabas, F., Zheng, J., Deberardinis, R. J., Mahmoud, A. I., Olson, E. N., Schneider, J. W., Zhang, C. C. \& Sadek, H. A. 2010. The distinct metabolic profile of hematopoietic stem cells reflects their location in a hypoxic niche. Cell Stem Cell, 7, 380-90. Takubo, K., Nagamatsu, G., Kobayashi, Chiharu i., Nakamura-Ishizu, A., Kobayashi, H., Ikeda, E., Goda, N., Rahimi, Y., Johnson, Randall s., Soga, T., Hirao, A., Suematsu, M. \& Suda, T. 2013. Regulation of Glycolysis by Pdk Functions as a Metabolic Checkpoint for Cell Cycle Quiescence in Hematopoietic Stem Cells. Cell Stem Cell, 12, 49-61.

Tasdogan, A., Faubert, B., Ramesh, V., Ubellacker, J. M., Shen, B., Solmonson, A., Murphy, M. M., Gu, Z., Gu, W., Martin, M., Kasitinon, S. Y., Vandergriff, T., Mathews, T. P., Zhao, Z., 
Schadendorf, D., Deberardinis, R. J. \& Morrison, S. J. 2020. Metabolic heterogeneity confers differences in melanoma metastatic potential. Nature, 577, 115-120.

Wang, Y. H., Israelsen, W. J., Lee, D., Yu, V. W., Jeanson, N. T., Clish, C. B., Cantley, L. C., Vander Heiden, M. G. \& Scadden, D. T. 2014. Cell-state-specific metabolic dependency in hematopoiesis and leukemogenesis. Cell, 158, 1309-23.

Washko, P. W., Welch, R. W., Dhariwal, K. R., Wang, Y. \& Levine, M. 1992. Ascorbic acid and dehydroascorbic acid analyses in biological samples. Analytical Biochemistry, 204, 1-14.

Xie, S. Z., Garcia-Prat, L., Voisin, V., Ferrari, R., Gan, O. I., Wagenblast, E., Kaufmann, K. B., Zeng, A. G. X., Takayanagi, S.-I., Patel, I., Lee, E. K., Jargstorf, J., Holmes, G., Romm, G., Pan, K., Shoong, M., Vedi, A., Luberto, C., Minden, M. D., Bader, G. D., Laurenti, E. \& Dick, J. E. 2019. Sphingolipid Modulation Activates Proteostasis Programs to Govern Human Hematopoietic Stem Cell Self-Renewal. Cell stem cell, 25, 639-653.e7.

Zheng, Y., Lin, T. Y., Lee, G., Paddock, M. N., Momb, J., Cheng, Z., Li, Q., Fei, D. L., Stein, B. D., Ramsamooj, S., Zhang, G., Blenis, J. \& Cantley, L. C. 2018. Mitochondrial One-Carbon Pathway Supports Cytosolic Folate Integrity in Cancer Cells. Cell, 175, 1546-1560.e17.

\section{Acknowledgements}

S.J.M. is a Howard Hughes Medical Institute (HHMI) Investigator, the Mary McDermott Cook Chair in Pediatric Genetics, the Kathryn and Gene Bishop Distinguished Chair in Pediatric Research, the director of the Hamon Laboratory for Stem Cells and Cancer, and a Cancer Prevention and Research Institute of Texas Scholar. A.W.D was supported by a Ruth L.

Kirschstein NRSA Fellowship. A.T. was supported by the Leopoldina Fellowship Program (LPDS 2016-16) of the German National Academy of Sciences and the Fritz Thyssen Foundation. This work was also funded by the National Institutes of Health (DK11875 and CA228608) and the Cancer Prevention and Research Institute of Texas (RP180778). Jian Xu 
provided the AML and ALL cell lines used in this study. The BioHPC high performance computing cloud at UTSW was used for data analysis and storage. The Moody Foundation Flow Cytometry Core was used for all flow cytometry. The graphic in Figure 4B was created using BioRender (BioRender.com).

\section{Contributions}

A.W.D., T.P.M., M.A. and S.J.M conceived the project, designed, and interpreted experiments. A.W.D. performed most of the experiments. T.P.M. developed the liquid chromatography and mass spectrometry methods, including implementation of HILIC and metabolite library construction. Z.Z. developed the low cell number metabolomics data analysis methods and performed all statistical analyses. M.S.M-S. analyzed mass spectrometry data and assisted T.P.M. with LC-MS method development. J.M.U. performed melanoma xenograft experiments.

A.T. assisted A.W.D. with the design and execution of methotrexate experiments. A.W.D., T.P.M., and S.J.M wrote the manuscript.

\section{Corresponding Author}

Correspondence and requests for materials should be addressed to Sean J. Morrison.

(Sean.Morrison@UTSouthwestern.edu) or Thomas P, Matthews

\section{(Thomas.Mathews@UTSouthwestern.edu).}

\section{Author Declarations}

S.J.M. is an advisor for Frequency Therapeutics and Protein Fluidics as well as a stockholder in G1 Therapeutics and Mereo Biopharma.

\section{FIGURE LEGENDS}

Figure 1: Sample processing and chromatography parameters. 
(A) The number of metabolites identified with high confidence spectral database matching in WBM samples after reverse phase versus HILIC $(n=3$ replicates per group from a single experiment). (B) Average peak intensities in sheath fluid background samples after drying with a standard vacuum concentrator, a vacuum concentrator housed in a positive pressure HEPAfiltered clean hood, or with no drying ( $n=5$ replicates per treatment from one experiment). (C) Number of metabolites significantly above sheath fluid background in 10,000 sorted WBM cells after drying with a standard vacuum concentrator, a vacuum concentrator housed in a clean hood, or with no drying $(n=5$ replicates per treatment from one experiment; fold change $>2$, FDR $<0.05$ by GLM). (D) Metabolites detected in 100,000 WBM cells extracted with $80 \%$ acetonitrile in water (ACN), $80 \%$ methanol in water $(\mathrm{MeOH})$, or $40 \% \mathrm{ACN}$ plus $40 \% \mathrm{MeOH}$ in water (Mix) ( $n=3$ replicates per treatment from one experiment; fold change $>2$, FDR $<0.05$ by GLM). (E) Overlap in metabolites detected with each extraction solvent $(n=3$ replicates per treatment from one experiment). (F) Number of metabolites significantly above background in 10,000 WBM cells sorted using a 70 or $100 \mu \mathrm{m}$ nozzle, and $0.5 x$ or $1.0 x$ PBS sheath fluid $(n=5$ replicates per treatment in each of 3 independent experiments; fold change $>2$, FDR $<0.05$ by GLM). (G) Number of metabolites significantly above background in 10,000 sorted WBM cells or 10,000 to 100,000 pipetted WBM cells $(n=5$ replicates per treatment in each of 3 independent experiments; fold change $>2$, FDR $<0.05$ by GLM). (H-K) $\mathbf{H}$, Principal component analysis of 10,000 sorted or pipetted HNT-34 AML (AML) cells or DND-41 T-ALL (ALL) cells (one experiment with $n=8$ replicates per treatment). (I) Metabolites that significantly changed between AML and ALL cells in sorted versus pipetted samples. (J) Correlation between Log2 fold changes (in AML versus ALL cells) in sorted versus pipetted samples for all detected metabolites. (K) Correlation between Log2 fold changes (in AML versus ALL cells) in sorted versus pipetted samples for metabolites that significantly differed between sorted AML versus ALL cells. (L-P) I, Number of metabolites observed above background in 10,000 pipetted WBM cell samples at various time points after the death of the mouse (one experiment with $n=5$ 
replicates per time point). (M) Number of metabolites that significantly increased or decreased at each time point relative to the 5 minute time point (fold change $>2$, FDR $<0.01$ by GLM; the identities of the metabolites are shown in Table 1). (N) Log2 transformed intensity values for all metabolites in the 5 minute versus 120 minute samples. (0) Non-transformed intensity values for all metabolites in the 5 minute versus 120 minute samples. (P) Non-transformed intensity values for metabolites $<1 \times 10^{8}$ in the 5 minute versus 120 minute samples. Statistical significance was assessed using paired t-test $(A)$, Kolmogorov-Smirnov test $(B)$ followed by Holm-Sidak's multiple comparisons adjustment, repeated measures one-way ANOVA followed by Tukey's (D) or Dunnett's $(G)$ multiple comparisons adjustment, repeated measures two-way ANOVA followed by Sidak's multiple comparisons adjustment $(F)$, or Spearman correlation analysis (J, K, N-P). All statistical tests were two-sided. Data represent mean \pm SD.

Figure 2. Metabolic differences between HSC/MPPs and WBM cells.

(A) Metabolites significantly (by two-sided paired t-test) above background in 10,000 sorted HSC/MPPs or WBM cells $(n=3-7$ replicates per treatment in each of 4 independent experiments; fold change $>2$, FDR $<0.05$ by GLM). (B) Heat map of metabolites that were significantly depleted (left) or enriched (right) in HSC/MPPs as compared to WBM cells (FDR < 0.01 , fold change $>2.5$, the $z$ score reflects the number of standard deviations from the mean for each metabolite in each sample, signal intensity values for each metabolite were log2 transformed before calculating z scores). Data in (A) represent mean $\pm S D$.

\section{Figure 3. Metabolic differences between methotrexate-treated and control HSCs or} circulating melanoma cells and primary tumors.

(A-D) Bone marrow cellularity (A) and the frequencies of CD $150^{+} \mathrm{CD} 48^{-} \mathrm{Lin}^{-} \mathrm{Sca} 1^{+} \mathrm{C}-\mathrm{kit}^{+} \mathrm{HSCs}$ (B), CD150-CD48-Lin'Sca $1^{+} \mathrm{C}-\mathrm{kit}^{+}$MPPs (c), and Lin $^{-}$Sca $1^{+} \mathrm{C}-\mathrm{kit}^{+}$cells (D) in femurs and tibias from mice treated with methotrexate or vehicle control $(n=5$ mice per treatment from 2 
independent experiments). (E) Percentage of nucleated blood cells that were donor-derived after competitive transplantation of bone marrow cells from methotrexate-treated versus control mice into irradiated recipients (two independent experiments). (F) AICAR levels in HSC/MPPs from mice treated with methotrexate or vehicle (11 control samples and 9 MTX samples from 4 independent experiments). (G) Metabolites detected above background in primary tumor cells or circulating melanoma cells $(n=3$ or 4 replicates per treatment in one experiment; fold change > 2, FDR $<0.05$ by GLM). (H-K) Levels of the purines IMP (H), XMP (I), GMP (J), and AMP (K) in primary tumor and circulating melanoma cells. Statistical significance was assessed by t-test (A), repeated measures two-way ANOVA (B-D) or mixed effects analysis (E) followed by Sidak's multiple comparisons adjustment. All tests were two-sided. Data represent mean \pm SD.

Figure 4: Metabolomic profiling of hematopoietic stem cells (HSCs) isolated by flow cytometry. (A) Overview of the method. (B) Metabolites detected above background in 10,000 HSCs/MPPs in this study (green numbers, 159 metabolites total) as compared to our prior study using a different method(Agathocleous et al., 2017) (blue numbers, 57 metabolites total). These data are from a single experiment, representative of 4 independent experiments. Metabolites detected above background were calculated by comparing 3 WBM or 3 HSC/MPP samples to 3 sheath fluid blanks (fold change $>2$, FDR $<0.05$ by Generalized Linear Models adjusted for multiple comparisons using the False Discovery Rate (FDR) method).

\section{Supplementary Figures}

Figure 2 - figure supplement 1. Metabolites that were detected as being different between HSCs/MPPs and WBM cells using the Agathocleous et al. metabolomics method (Agathocleous et al., 2017) versus the new method. Metabolites identified by Agathocleous et al. as (A) enriched or (B) depleted in HSC/MPPs as compared to WBM cells (FDR $<0.01$ by GLM) (the new method data are from the experiments shown in Figure 2). 
Figure 2 - figure supplement 2. Glycerophospholipids are enriched in HSC/MPPs as compared to WBM cells. (A) Schematic of glycerophospholipid metabolism and (B) list of phosphatidylcholines (PC), phosphatidylethanolamines (PE), phosphatidylserines (PS), LysoPCs, and Lyso-PEs detected in 10,000 HSCs/MPPs or WBM cells by our method. Red metabolites were enriched in HSC/MPPs and blue metabolites were depleted in HSC/MPPs. Purple metabolites were detected but not significantly changed. Black metabolites were not detected ( $n=11$ replicates per cell population from a total of 4 independent experiments).

Figure 3 - figure supplement 1. Metabolites that differed between circulating melanoma cells and primary subcutaneous tumors in xenografted mice. The heatmap shows z scores of all the metabolites that significantly $(P<0.05$ by GLM) differed between 10,000 cell aliquots of circulating melanoma cells and primary subcutaneous tumor cells. The $z$ score reflects the number of standard deviations from the mean for each metabolite in each sample $(n=4$ replicates per cell population from one experiment; signal intensity values for each metabolite were log2 transformed before calculating z scores).

Figure 3 - figure supplement 2. Flow cytometry gating strategies. (A) Flow cytometry gating

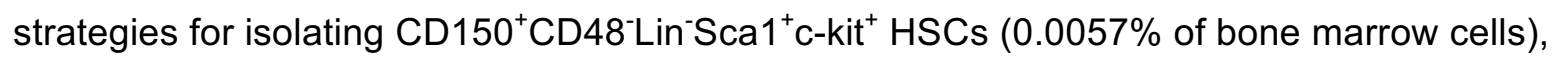
CD150-CD48-Lin`Sca ${ }^{+}{ }^{-} \mathrm{C}-\mathrm{kit}^{+}$MPPs $(0.0047 \%$ of bone marrow cells $)$, and Lin $^{-}$Sca $1^{+} \mathrm{c}-\mathrm{kit}^{+}$cells ( $0.19 \%$ of bone marrow cells). (B) Flow cytometry gating strategy for isolating live $\mathrm{HLA}^{+}$DsRed ${ }^{+} \mathrm{mTer119}{ }^{-m} \mathrm{mCD}^{2} 5^{-m} \mathrm{mCD}^{-} 1^{-}$human melanoma cells from mechanically dissociated subcutaneous tumors from xenografted NSG mice (all melanomas were tagged with stable DsRed expression). (C) Flow cytometry gating strategy for isolating live $\mathrm{HLA}^{+} \mathrm{DsRed}^{+} \mathrm{mTer119}$ mCD45 mCD31' circulating melanoma cells from the blood of xenografted NSG mice. 
Figure 1 - supplementary table 1. Metabolites enriched or depleted at different time

\section{points after animal death as compared to 5 minutes after animal death}

\begin{tabular}{|l|c|c|}
\hline \multicolumn{3}{|c|}{ 15 $\mathbf{~ m}$ in vs $\mathbf{5}$ in } \\
\hline \multicolumn{1}{|c|}{ Metabolite } & FC & FDR \\
\hline Carnitine (3-hydroxy-14:0) & 2.34 & 0.0040 \\
\hline Carnitine (3-hydroxy-C16:0) & 2.25 & 0.0001 \\
\hline
\end{tabular}

\begin{tabular}{|l|c|c|}
\hline \multicolumn{3}{|c|}{30 m in vs $\mathbf{5}$ min } \\
\hline \multicolumn{1}{|c|}{ Metabolite } & FC & FDR \\
\hline Guanosine monophosphate & 3.42 & 0.0092 \\
\hline Carnitine (3-hydroxy-14:0) & 3.29 & 0.0000 \\
\hline Carnitine (3-hydroxy-C16:0) & 3.08 & 0.0000 \\
\hline Adenosine monophosphate & 2.78 & 0.0010 \\
\hline Hypoxanthine & 2.50 & 0.0052 \\
\hline 2'_3'-CyclicCMP & 2.42 & 0.0054 \\
\hline
\end{tabular}

\begin{tabular}{|l|c|c|}
\hline \multicolumn{3}{|c|}{$\mathbf{6 0}$ m in vs $\mathbf{5}$ $\mathbf{~ i n ~}$} \\
\hline Metabolite & FC & FDR \\
\hline Carnitine (3-hydroxy-C16:0) & 3.65 & 0.0000 \\
\hline Carnitine (3-hydroxy-14:0) & 3.64 & 0.0052 \\
\hline Carnitine (3-hydroxy-8:0) & 3.61 & 0.0000 \\
\hline Hypoxanthine & 3.25 & 0.0073 \\
\hline 2'_3'-CyclicCMP & 3.25 & 0.0003 \\
\hline Adenosine & 3.23 & 0.0002 \\
\hline Adenosine monophosphate & 2.87 & 0.0000 \\
\hline Inosine & 2.83 & 0.0006 \\
\hline Carnitine (16:0) & 2.74 & 0.0000 \\
\hline Nicotinamide & 2.36 & 0.0000 \\
\hline Carnitine (16:1) & 2.13 & 0.0000 \\
\hline Lyso-PC (18:2) & 2.01 & 0.0000 \\
\hline
\end{tabular}

\begin{tabular}{|c|c|c|}
\hline \multicolumn{3}{|l|}{$120 \mathrm{~m}$ in vs $5 \mathrm{~m}$ in } \\
\hline Metabolite & FC & FDR \\
\hline arg-ala & 11.36 & 0.0017 \\
\hline Hypoxanthine & 8.43 & 0.0000 \\
\hline tyrosylarginine & 7.23 & 0.0027 \\
\hline Pentose-phosphates & 6.92 & 0.0017 \\
\hline Inosine & 6.48 & 0.0000 \\
\hline Guanosine monophosphate & 4.65 & 0.0008 \\
\hline Hydroxyoctanoic acid $(\mathrm{C} 8,-\mathrm{OH})$ & 4.48 & 0.0035 \\
\hline Ecosadienoic acid & 3.97 & 0.0017 \\
\hline Adenosine & 3.85 & 0.0000 \\
\hline Uridine Monophosphate & 3.59 & 0.0030 \\
\hline 2'_3'-CyclicCMP & 3.59 & 0.0001 \\
\hline Carnitine (3-hydroxy-C16:0) & 3.15 & 0.0000 \\
\hline Carnitine (3-hydroxy-14:0) & 3.11 & 0.0000 \\
\hline Carnitine (3-hydroxy-8:0) & 3.03 & 0.0092 \\
\hline Adenosine monophosphate & 3.03 & 0.0002 \\
\hline Carnitine $(16: 0)$ & 2.95 & 0.0000 \\
\hline Nicotinamide & 2.48 & 0.0000 \\
\hline hydroxydecanoic Acid (C10:0, $-\mathrm{OH})$ & 2.45 & 0.0046 \\
\hline Choline & 2.13 & 0.0000 \\
\hline NAD+ & 0.46 & 0.0017 \\
\hline CyclicADP-ribose & 0.46 & 0.0084 \\
\hline Lyso-PC (22:4) & 0.38 & 0.0000 \\
\hline Lyso-PC (18:1) & 0.36 & 0.0000 \\
\hline Lyso-PC (16:0) & 0.35 & 0.0000 \\
\hline Lyso-PC (18:2) & 0.21 & 0.0000 \\
\hline
\end{tabular}

\begin{tabular}{|c|c|c|}
\hline \multicolumn{3}{|l|}{$180 \mathrm{~m}$ in vs $5 \mathrm{~m}$ in } \\
\hline Metabolite & FC & FDR \\
\hline arg-ala & 16.17 & 0.0004 \\
\hline Pentose-phosphates & 14.00 & 0.0000 \\
\hline Hypoxanthine & 12.32 & 0.0000 \\
\hline Inosine & 8.57 & 0.0000 \\
\hline tyrosylarginine & 8.44 & 0.0012 \\
\hline Guanosine monophosphate & 7.63 & 0.0000 \\
\hline Uridine Monophosphate & 6.03 & 0.0001 \\
\hline Xanthine & 5.02 & 0.0070 \\
\hline 2'_3'-CyclicCMP & 4.46 & 0.0000 \\
\hline Adenosine monophosphate & 3.98 & 0.0000 \\
\hline Ecosadienoic acid & 3.80 & 0.0022 \\
\hline Adenosine & 3.72 & 0.0000 \\
\hline Carnitine (3-hydroxy-C16:0) & 3.03 & 0.0000 \\
\hline Adenosine diphosphate ribose & 2.90 & 0.0030 \\
\hline Nicotinamide & 2.87 & 0.0000 \\
\hline Carnitine (16:0) & 2.61 & 0.0000 \\
\hline Carnitine (3-hydroxy-14:0) & 2.34 & 0.0007 \\
\hline Choline & 2.04 & 0.0000 \\
\hline Platelet-activating factor & 0.43 & 0.0000 \\
\hline Lyso-PC (22:4) & 0.34 & 0.0000 \\
\hline Lyso-PC (18:1) & 0.31 & 0.0000 \\
\hline Lyso-PC (16:0) & 0.30 & 0.0000 \\
\hline Lyso-PC (18:2) & 0.16 & 0.0000 \\
\hline Lyso-PC (17:0) & 0.11 & 0.0001 \\
\hline
\end{tabular}

\begin{tabular}{|c|c|c|}
\hline \multicolumn{3}{|l|}{$240 \mathrm{~m}$ in vs $5 \mathrm{~min}$} \\
\hline Metabolite & FC & FDR \\
\hline Hypoxanthine & 18.20 & 0.0000 \\
\hline arg-ala & 13.03 & 0.0009 \\
\hline Inosine & 12.04 & 0.0000 \\
\hline tyrosylarginine & 8.94 & 0.0009 \\
\hline Pentose-phosphates & 8.35 & 0.0007 \\
\hline Xanthine & 7.14 & 0.0009 \\
\hline Eicosadienoic acid & 5.42 & 0.0002 \\
\hline Hydroxyoctanoic acid $(\mathrm{C} 8,-\mathrm{OH})$ & 5.07 & 0.0014 \\
\hline Guanosine monophosphate & 4.38 & 0.0009 \\
\hline Adenosine & 4.35 & 0.0000 \\
\hline Carnitine (3-hydroxy-8:0) & 3.65 & 0.0019 \\
\hline Uridine Monophosphate & 3.57 & 0.0026 \\
\hline 2'_3'-CyclicCMP & 3.54 & 0.0001 \\
\hline Carnitine (3-hydroxy-C16:0) & 3.29 & 0.0000 \\
\hline Nicotinamide & 3.25 & 0.0000 \\
\hline Carnitine (16:0) & 2.95 & 0.0000 \\
\hline hydroxydecanoic Acid (C10:0, $-\mathrm{OH})$ & 2.72 & 0.0014 \\
\hline Adenosine monophosphate & 2.54 & 0.0012 \\
\hline Choline & 2.41 & 0.0000 \\
\hline Carnitine (3-hydroxy-14:0) & 2.28 & 0.0009 \\
\hline Oleic acid & 2.06 & 0.0073 \\
\hline Platelet-activating factor & 0.41 & 0.0000 \\
\hline NAD+ & 0.35 & 0.0000 \\
\hline CyclicADP-ribose & 0.33 & 0.0003 \\
\hline Lyso-PC (16:0) & 0.26 & 0.0000 \\
\hline Lyso-PC (22:4) & 0.26 & 0.0000 \\
\hline Lyso-PC (18:1) & 0.26 & 0.0000 \\
\hline Adenosine triphosphate (ATP) & 0.17 & 0.0086 \\
\hline Lyso-PC (18:2) & 0.13 & 0.0000 \\
\hline Lyso-PC $(17: 0)$ & 0.04 & 0.0000 \\
\hline
\end{tabular}


bioRxiv preprint doi: https://doi.org/10.1101/2020.08.11.246900; this version posted August 12, 2020. The copyright holder for this preprint

(which was not certified by peer review) is the author/funder. All rights reserved. No reuse allowed without permission.

Figure 2 - supplementary table 1. Metabolites enriched or depleted in sorted HSC/MPPs

\section{as compared to WBM cells}

\begin{tabular}{|l|c|c|}
\hline \multicolumn{3}{|c|}{ Depleted in HSC/MPP vs WBM } \\
\hline \multicolumn{1}{|c|}{ Metabolite } & FC & FDR \\
\hline PC (18:0/18:0) & 0.0030 & 0.0000 \\
\hline Uric acid & 0.018 & 0.0000 \\
\hline N-Ribosylnicotinamide & 0.036 & 0.0000 \\
\hline Acetyl-hexosamine-sulfate & 0.067 & 0.0000 \\
\hline 2'_3'-CyclicCMP $^{\prime}$ 'Crgininosuccinic acid & 0.077 & 0.0000 \\
\hline Inosine-5'-monophosphate & 0.081 & 0.0000 \\
\hline DL-Stachydrine & 0.10 & 0.0000 \\
\hline 2-(alpha-D-Mannosyl)-3-phosphoglycerate & 0.11 & 0.0000 \\
\hline AICAR & 0.12 & 0.0000 \\
\hline PS (38:6) & 0.12 & 0.0000 \\
\hline 3-Hydroxycarnitine (18:1) & 0.14 & 0.0000 \\
\hline PS (34:2) & 0.17 & 0.0000 \\
\hline Cholesteryl-acetate & 0.18 & 0.0000 \\
\hline DL-Carnitine & 0.18 & 0.0007 \\
\hline Isoleucine/ Leucine & 0.21 & 0.0000 \\
\hline PC (36:4) & 0.24 & 0.0023 \\
\hline Propionylcarnitine & 0.25 & 0.0000 \\
\hline N6,N6,N6-Trimethyl-L-lysine & 0.26 & 0.0000 \\
\hline PI (32:0) & 0.27 & 0.0000 \\
\hline Lyso-PC (18:0) & 0.29 & 0.0000 \\
\hline Carnitine (16:1) & 0.31 & 0.0013 \\
\hline Glutamine & 0.32 & 0.0000 \\
\hline Carnitine (4:0) & 0.33 & 0.0000 \\
\hline Acetylcholine & 0.36 & 0.0000 \\
\hline Aminolevulinic Acid & 0.37 & 0.0000 \\
\hline 3-Dehydroxycarnitine & 0.38 & 0.0016 \\
\hline Carnitine (18:1) & 0.39 & 0.0000 \\
\hline Guanidine acetic acid & 0.42 & 0.0000 \\
\hline Carnitine (14:0) & 0.43 & 0.0000 \\
\hline Carnitine (3-hydroxy-C16:0) & 0.44 & 0.0000 \\
\hline Carnitine (3-hydroxy-14:0) & 0.44 & 0.0012 \\
\hline SM (d18:1/16:1) & 0.45 & 0.0054 \\
\hline Carnitine (16:0) & 0.47 & 0.0000 \\
\hline Carnitine (12:0) & 0.48 & 0.0000 \\
\hline D-(+)-Malic acid & 0.48 & 0.0015 \\
\hline PE (40:5) & 0.51 & 0.0001 \\
\hline N-Acetylaspartic acid & 0.55 & 0.0000 \\
\hline PS (40:7) & 0.57 & 0.0000 \\
\hline L-Glutathione oxidized & 0.0064 \\
\hline UDP-Hexose & 0.0035 \\
\hline Taurine & 0.0000 \\
\hline Uridine5'-diphosphate(UDP) & 0.0000 \\
\hline FA (8:0) & 0.0000 \\
\hline PE (36:6) & 0.002 \\
\hline PS (40:6) & 0.67 \\
\hline
\end{tabular}

\begin{tabular}{|c|c|c|}
\hline \multicolumn{3}{|c|}{ Enriched in HSC/MPP vs WBM } \\
\hline Metabolite & FC & FDR \\
\hline SM (d18:1/18:1) & 45 & 0.0000 \\
\hline Glycerylphosphorylcholine & 29 & 0.0000 \\
\hline Glycerophosphorylethanolamine & 22 & 0.0000 \\
\hline $\mathrm{PE}(38: 1)$ & 19 & 0.0000 \\
\hline Hexoses & 16 & 0.0000 \\
\hline $\mathrm{PC}(44: 8)$ & 14 & 0.0000 \\
\hline Lyso-PC (22:4) & 9.7 & 0.0000 \\
\hline Cysteinylglycine(Cys-Gly) & 7.4 & 0.0000 \\
\hline Glycerol1_2-cyclicphosphate & 5.8 & 0.0001 \\
\hline SM (d18:1/18:0) & 5.7 & 0.0000 \\
\hline L-Tyrosine & 5.2 & 0.0028 \\
\hline Adenosine diphosphate ribose & 4.9 & 0.0002 \\
\hline P-DMEA & 4.6 & 0.0000 \\
\hline Choline & 4.5 & 0.0000 \\
\hline Cholinephosphate & 4.3 & 0.0000 \\
\hline UTP & 3.8 & 0.0035 \\
\hline Valyllysine & 3.7 & 0.0000 \\
\hline S-Methyl-thioadenosine & 3.6 & 0.0001 \\
\hline Deoxy-heptulose-7-phosphate & 3.6 & 0.0044 \\
\hline Lyso-PC (18:1) & 3.5 & 0.0000 \\
\hline $\mathrm{PC}(40: 5)$ & 3.1 & 0.0000 \\
\hline Dipthamide & 2.8 & 0.0008 \\
\hline S-Adenosylmethionine & 2.7 & 0.0000 \\
\hline Cytidine 5'-diphosphocholine & 2.5 & 0.0007 \\
\hline O-Phosphorylethanolamine & 2.4 & 0.0000 \\
\hline $\mathrm{PC}(40: 4)$ & 2.4 & 0.0000 \\
\hline $\mathrm{PC}(36: 1)$ & 2.3 & 0.0000 \\
\hline Hydroxypyruvate & 2.3 & 0.0010 \\
\hline D-Saccharicacid & 2.2 & 0.0001 \\
\hline $\mathrm{PC}(34: 1)$ & 2.2 & 0.0000 \\
\hline $\mathrm{PC}(40: 8)$ & 2.1 & 0.0000 \\
\hline SM (d18:1/22:0) & 2.0 & 0.0000 \\
\hline Lyso-PE (20:4) & 1.9 & 0.0064 \\
\hline PE (36:3) & 1.9 & 0.0000 \\
\hline PE (38:3) & 1.8 & 0.0000 \\
\hline Adenosine monophosphate & 1.8 & 0.0010 \\
\hline PC (40:6) & 1.8 & 0.0000 \\
\hline Oleic acid & 1.8 & 0.0080 \\
\hline $\mathrm{PE}(34: 1)$ & 1.8 & 0.0000 \\
\hline $\mathrm{PC}(38: 4)$ & 1.8 & 0.0000 \\
\hline PE (38:5) & 1.8 & 0.0000 \\
\hline $\mathrm{PE}(36: 1)$ & 1.7 & 0.0000 \\
\hline $\mathrm{PE}(38: 4)$ & 1.7 & 0.0000 \\
\hline PS (36:1) & 1.6 & 0.0000 \\
\hline Adenosine triphosphate (ATP) & 1.6 & 0.0021 \\
\hline L-Glutathione (reduced) & 1.6 & 0.0026 \\
\hline $\mathrm{PC}(36: 5)$ & 1.5 & 0.0000 \\
\hline Cytidine monophosphate & 1.4 & 0.0010 \\
\hline Platelet-activating factor & 1.4 & 0.0099 \\
\hline $\mathrm{PC}(32: 2)$ & 1.3 & 0.0099 \\
\hline $\mathrm{PC}(36: 2)$ & 1.3 & 0.0009 \\
\hline PS (38:5) & 1.3 & 0.0044 \\
\hline
\end{tabular}




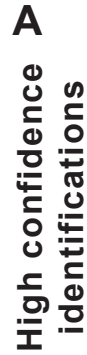

D

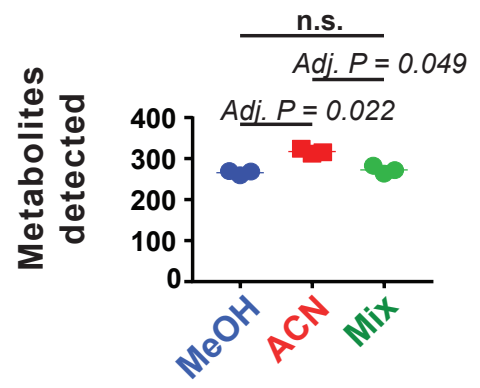

G
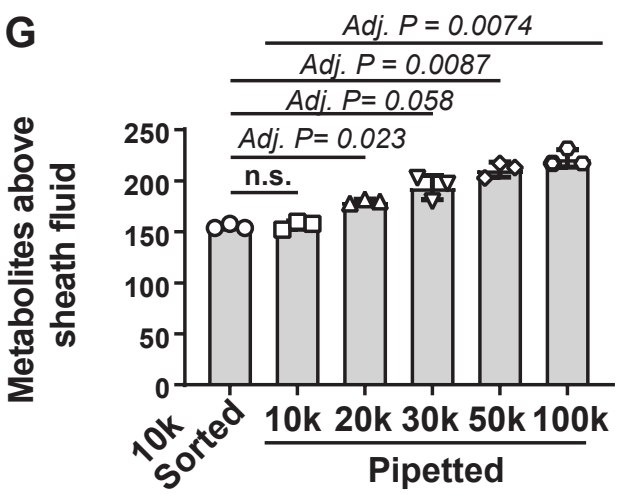

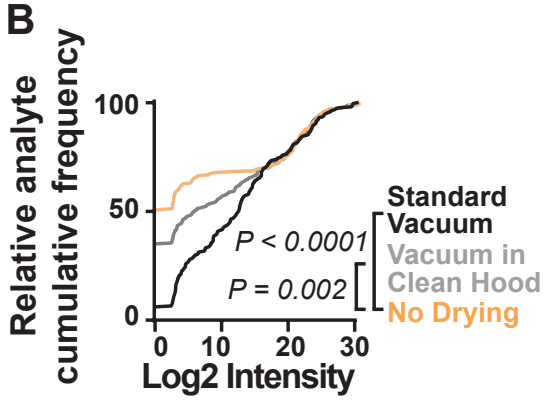

E

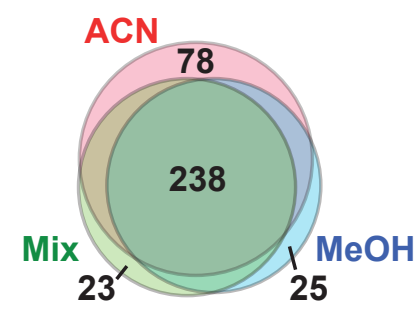

H

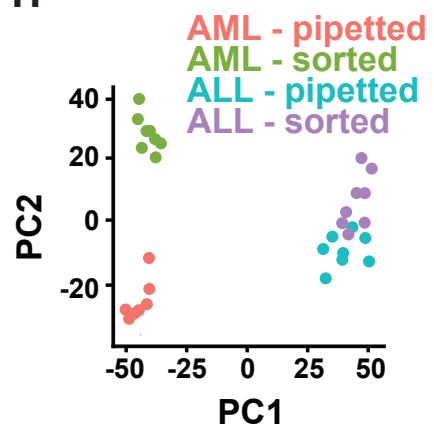

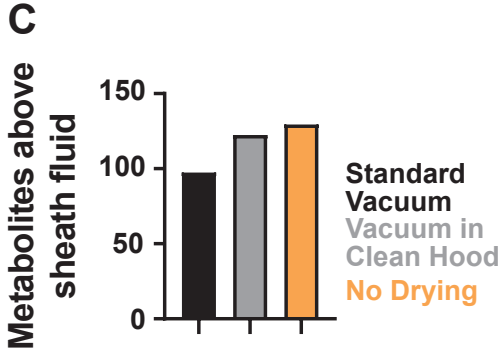

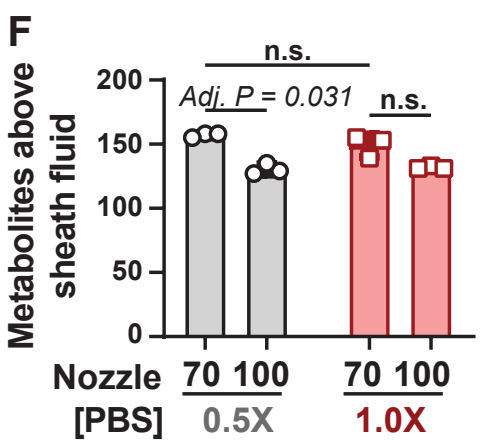

I

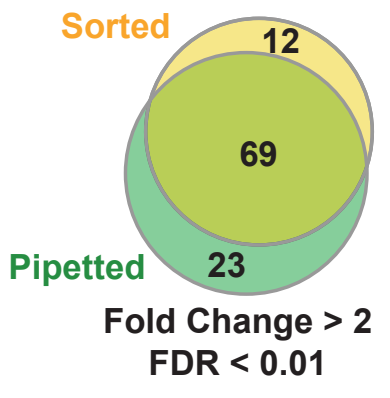

All Detected Metabolites

AML vs ALL

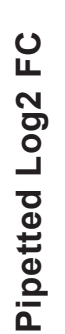

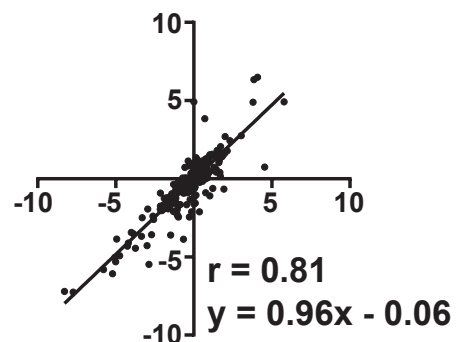

Sorted Log2 FC

M

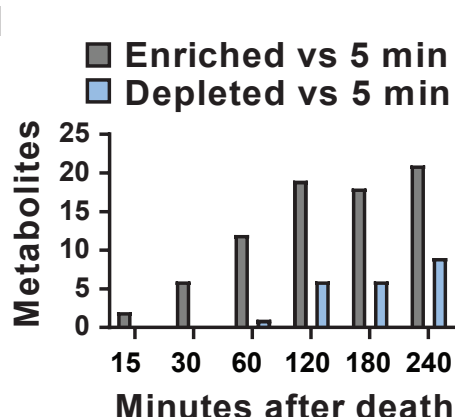

$\mathbf{N}$

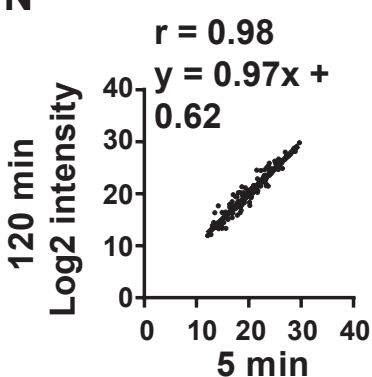

Log2 intensity

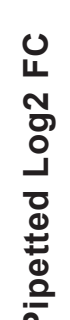

Sorted AML vs ALL

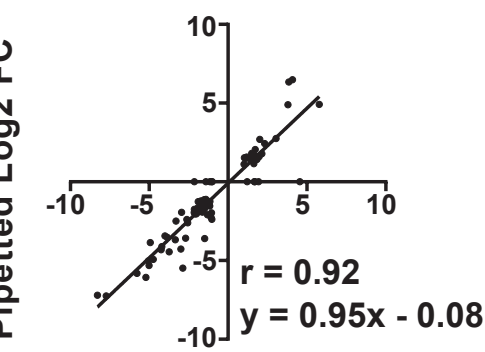

Sorted Log2 FC

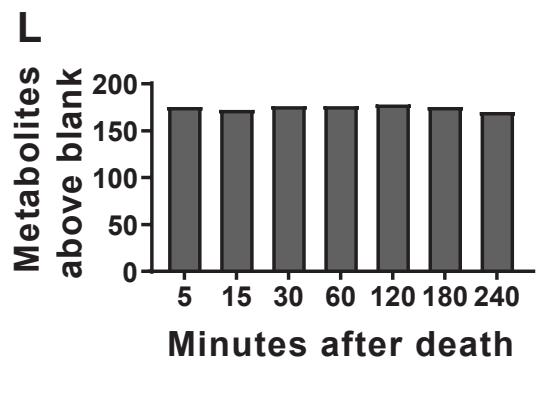

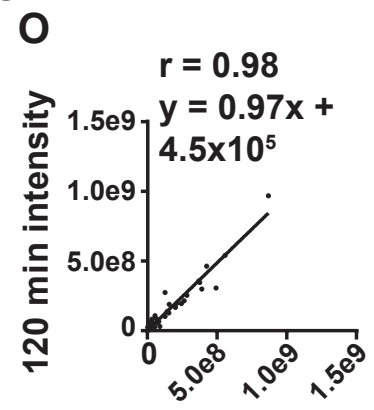

5 min intensity

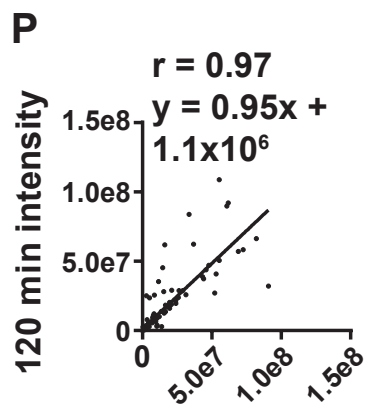

5 min intensity 
A

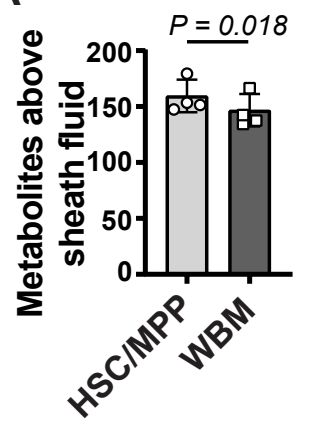

B

Depleted in HSC/MPPs WBM HSC/MPP

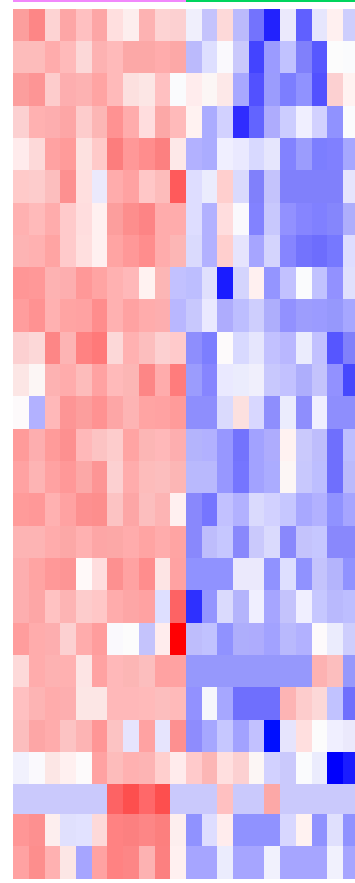

PI (32:0)

PS (34:2)

PS (38:6)

3-Hydroxycarnitine (18:1)

Carnitine (16:1)

AICAR

2-(a-D-Mannosyl)-3-phosphoglycerate IMP

Carnitine (4:0)

Stachydrine

Propionylcarnitine

PC (36:4)

Uric acid

3-Dehydroxycarnitine

Acetylcholine

Carnitine

PC (18:0/18:0)

N-Ribosylnicotinamide

Trimethyllysine

Glutamine

Isoleucine/Leucine

2',3'-CyclicCMP

Lyso-PC (18:0)

Cholesteryl-acetate

Aminolevulinic Acid

Acetyl-hexosamine-sulfate

Argininosuccinic acid

\section{Enriched in HSC/MPPs}
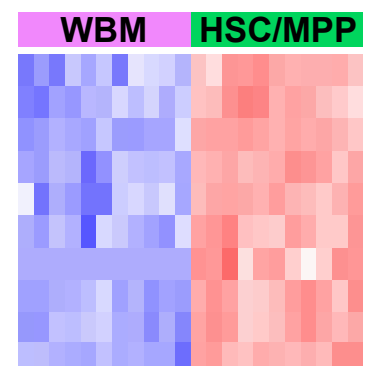

PE (38:1)

PC (40:5)

SM(d18:1/18:0)

Choline

Hexoses

Lyso-PC (18:1)

PC (44:8)

Cholinephosphate

P-DMEA

glycerophosphorylcholine SM (d18:1/18:0)

glycerophosphorylethanolamine

Glycerol1,2-cyclicphosphate

Lyso-PC (22:4)

Valyllysine

Cytidine 5'-diphosphocholine

Dipthamide

Deoxy-heptulose-7-phosphate

Adenosine diphosphate ribose

UTP

Cysteinylglycine

Tyrosine

SAM

S-Methyl-thioadenosine

Z-Score

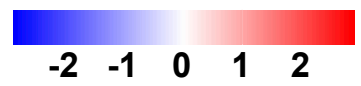


A

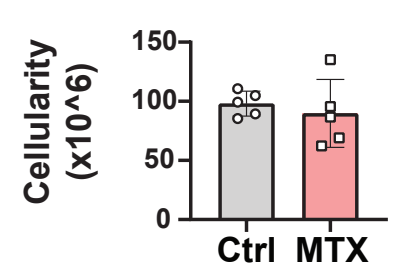

E

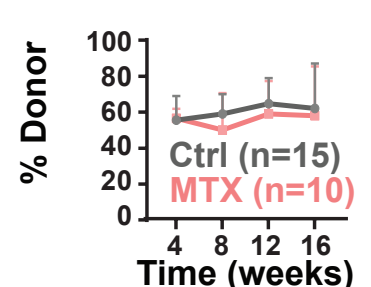

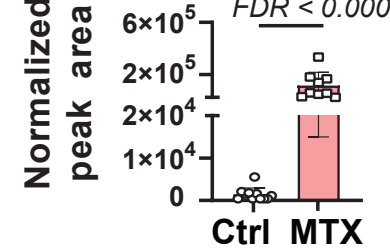

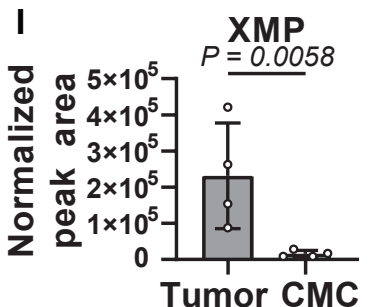

B

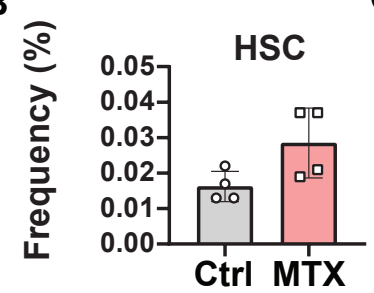

F

AlCAR 2000

C
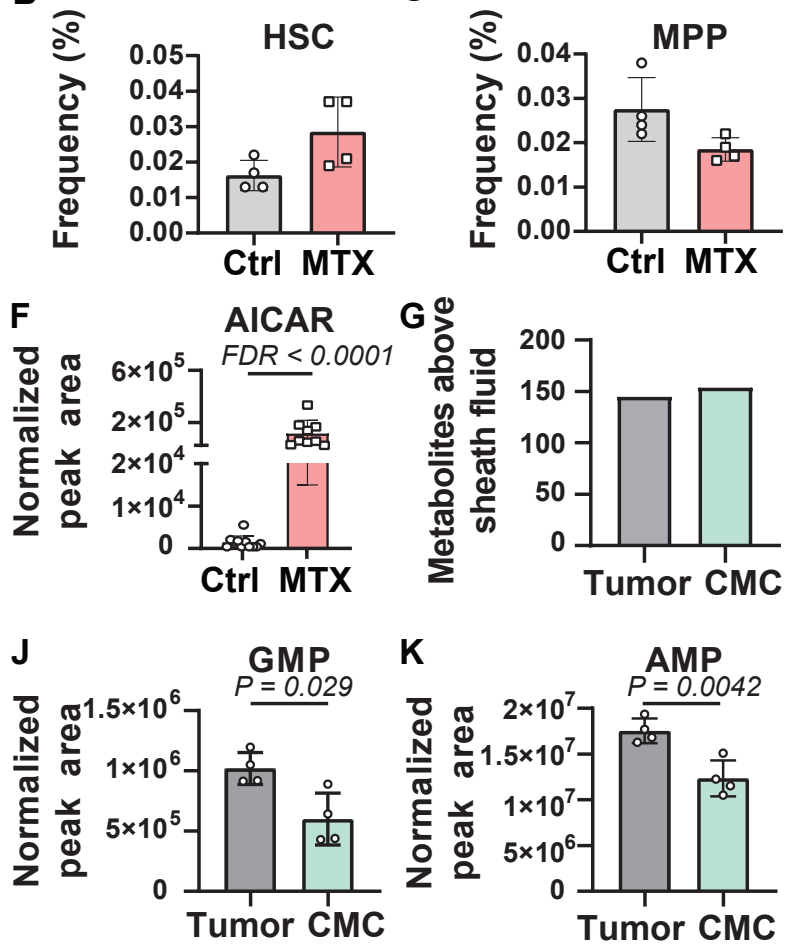

D
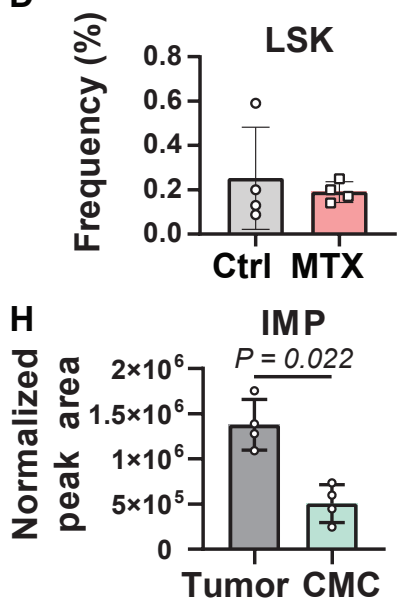
A Metabolites observed above background in initial method ${ }^{1}: 57$

Metabolites observed above background using new method (typical experiment): 159

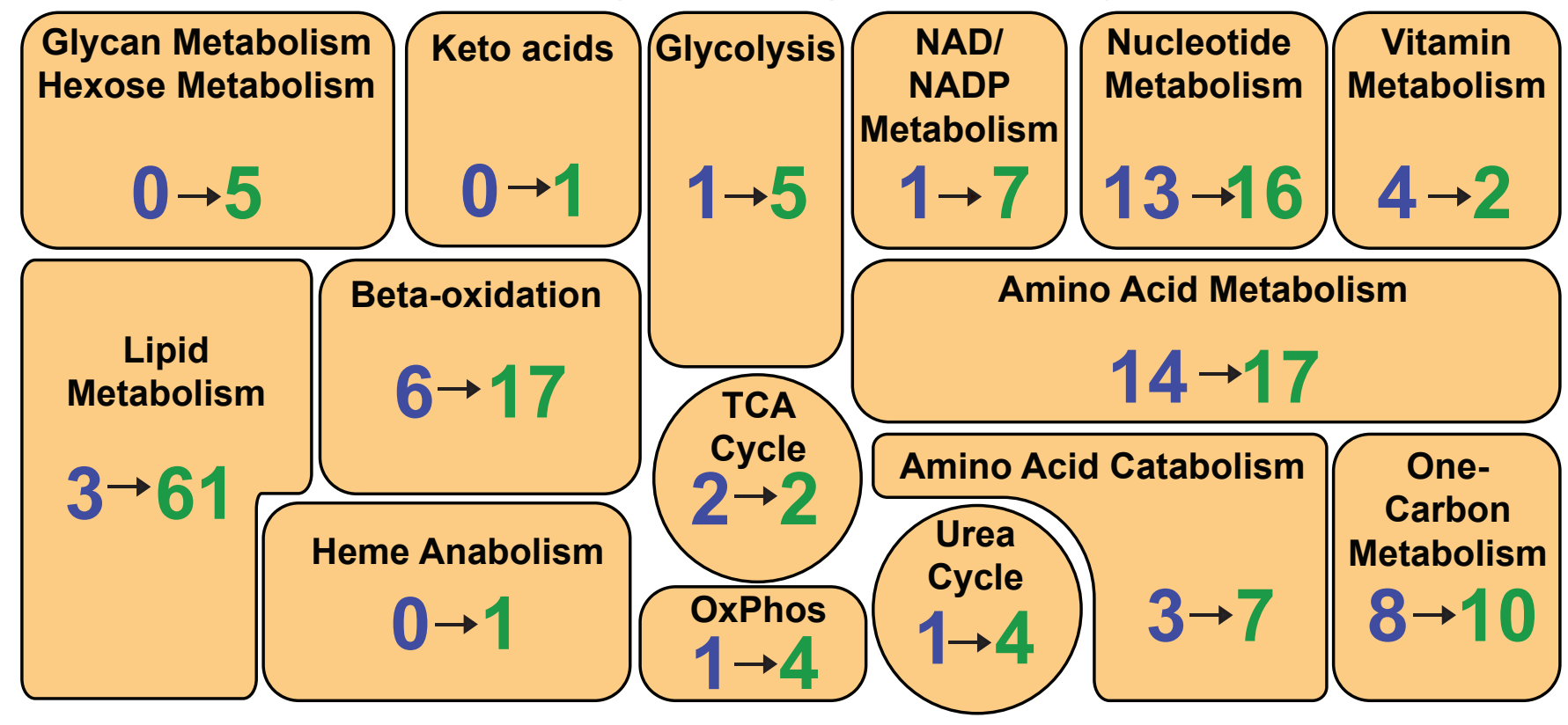

B

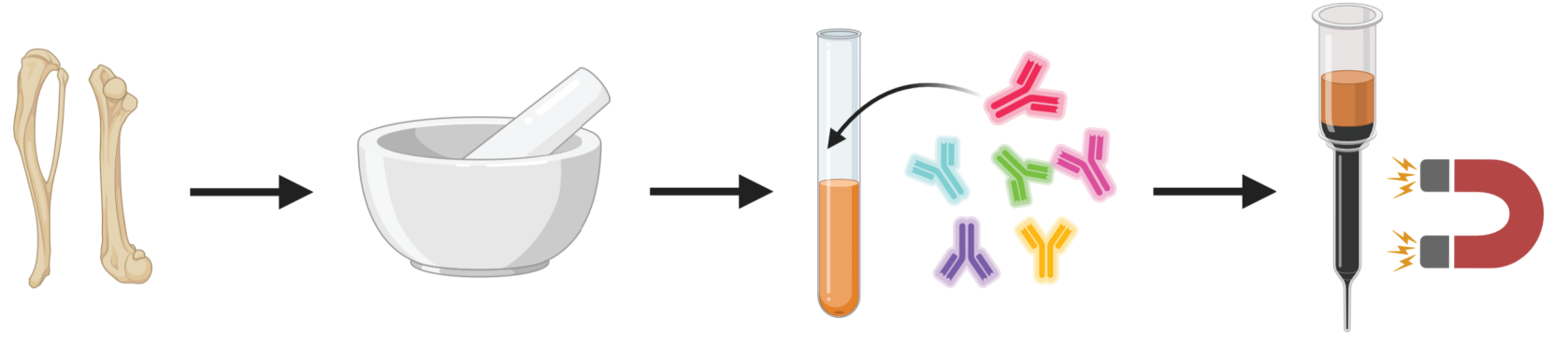

Isolate bones Crush bones on ice in rapidly at $4^{\circ} \mathrm{C} \quad 2 \mathrm{ml} \mathrm{HBSS}$ with cold mortar and pestle
Filter cells, add selection beads and antibody cocktail. No Centrifugation
Magnetic isolation of c-kit+ cells

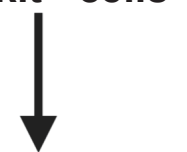

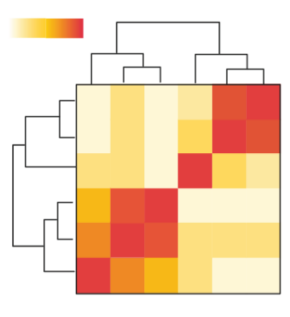

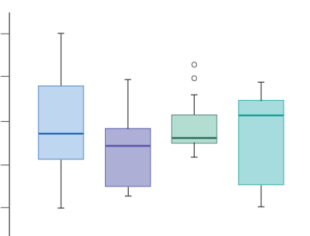

Determine metabolites with differential abundance using custom statistics pipeline

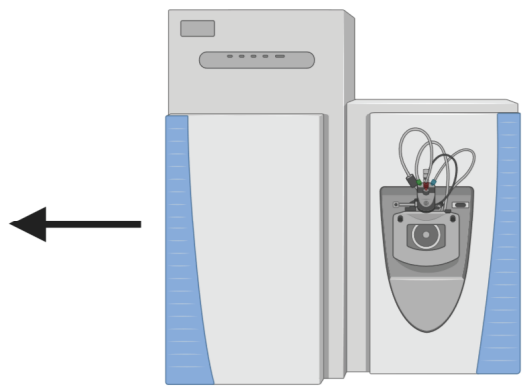

Quantitate 150-200 metabolites by LC-MS using HILIC

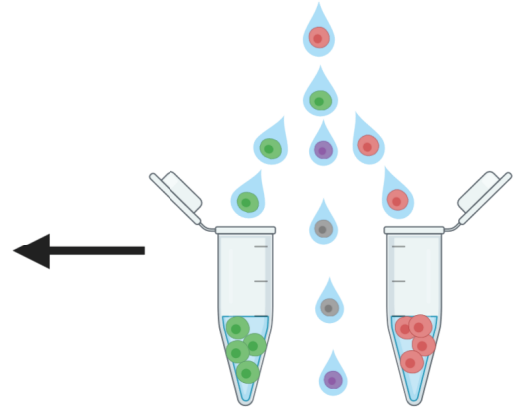

Sort 10,000 cells directly into ice cold acetonitrile. Use freshly prepared, ultra-clean, 0.5x PBS sheath fluid 


\section{Figure 2 - figure supplement 1}

A

Enriched in HSC/MPP v WBM

\begin{tabular}{|c|c|c|c|c|}
\hline & \multicolumn{2}{|c|}{ Agathocleous et al } & \multicolumn{2}{|c|}{ DeVilbiss et al } \\
\hline Metabolite & FC & FDR & FC & FDR \\
\hline glycerophosphorylcholine & 34 & $<0.0001$ & 29 & $<0.0001$ \\
\hline ascorbate & 18 & 0.0001 & \multicolumn{2}{|c|}{ n.d. (no EDTA added) } \\
\hline phosphocholine & 2.7 & $<0.0001$ & 4.3 & $<0.0001$ \\
\hline choline & 2.1 & $<0.0001$ & 4.5 & $<0.0001$ \\
\hline GSH & 2.0 & 0.002 & 1.6 & 0.0025 \\
\hline
\end{tabular}

B Depleted in HSC/MPP v WBM

\begin{tabular}{lcccr}
\hline & \multicolumn{2}{c}{ Agathocleous et al } & \multicolumn{2}{c}{ DeVilbiss et al } \\
\hline \multicolumn{1}{c}{ Metabolite } & FC & FDR & FC & \multicolumn{1}{c}{ FDR } \\
\hline glutamine & 0.18 & $<0.0001$ & 0.33 & $<0.0001$ \\
N-acetylaspartate & 0.30 & $<0.0001$ & 0.57 & $<0.0001$ \\
hypoxanthine & 0.34 & $<0.0001$ & 0.46 & 0.048 \\
carnitine & 0.36 & $<0.0001$ & 0.21 & $<0.0001$ \\
IMP & 0.36 & 0.0003 & 0.10 & $<0.0001$ \\
spermidine & 0.38 & 0.002 & n.d. & \\
acetylcholine & 0.42 & $<0.0001$ & 0.37 & $<0.0001$ \\
trimethyllysine & 0.48 & $<0.0001$ & 0.27 & $<0.0001$ \\
betaine & 0.50 & $<0.0001$ & n.d. & \\
glutamate & 0.64 & $<0.0001$ & 0.69 & 0.030 \\
taurine & 0.77 & 0.0001 & 0.64 & $<0.0001$
\end{tabular}


Figure 2 - figure supplement 2

A

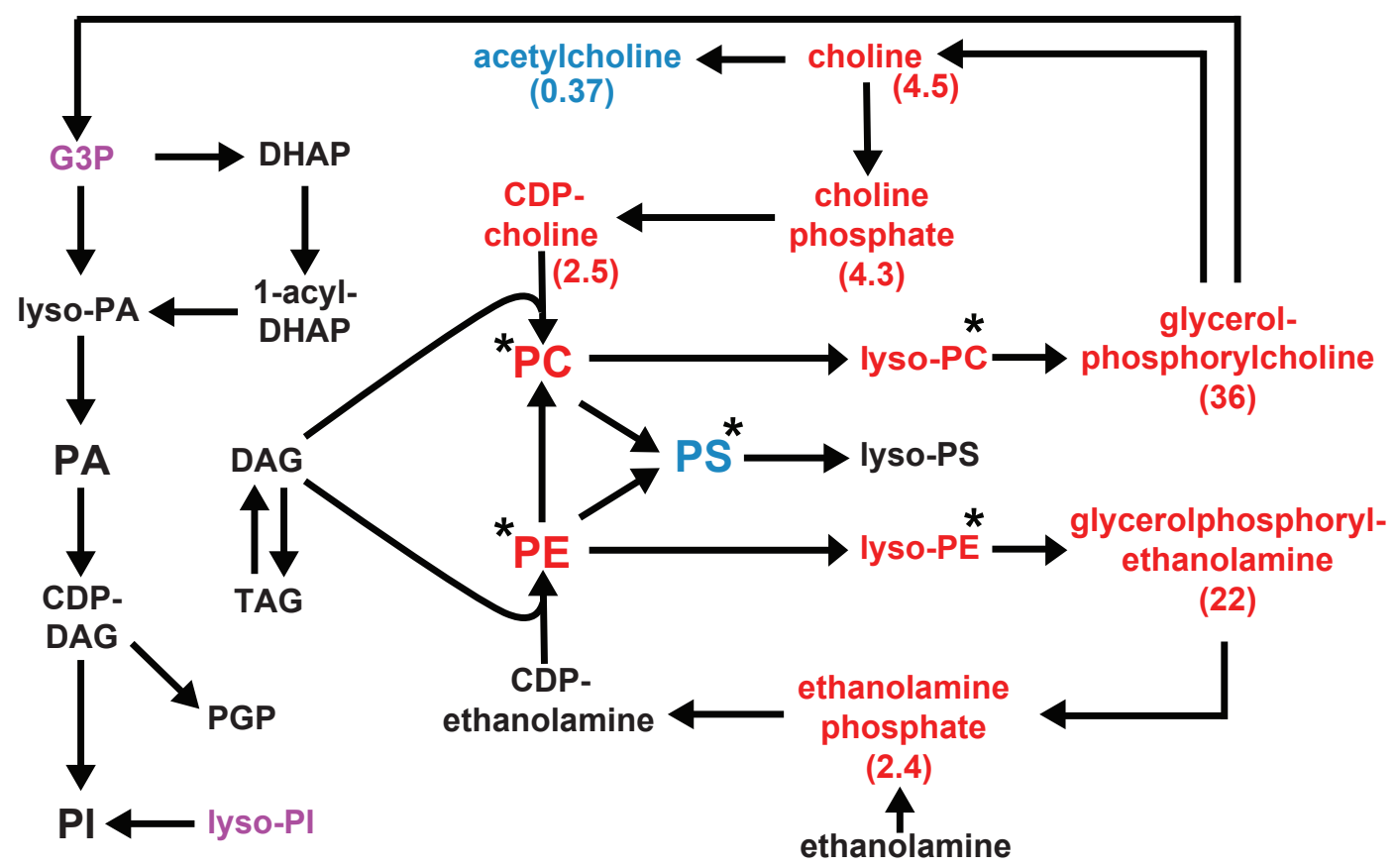

* Multiple metabolites detected in this class. See table.

Enriched in HSC/MPPs vs WBM Depleted in HSC/MPPs vs WBM Detected, No Change Not Detected

\begin{tabular}{lll} 
B & PC \\
\hline Metabolite & FC & FDR \\
\hline PC (32:0) & 1.1 & 0.26 \\
PC (32:1) & 1.2 & 0.015 \\
PC (32:2) & 1.3 & 0.0099 \\
PC (34:1) & 2.2 & 0.0000 \\
PC (34:2) & 1.1 & 0.11 \\
PC (36:1) & 2.3 & 0.0000 \\
PC (36:2) & 1.3 & 0.0008 \\
PC (36:4) & 0.25 & 0.0000 \\
PC (36:5) & 1.5 & 0.0000 \\
PC (38:4) & 1.8 & 0.0000 \\
PC (38:5) & 0.37 & 0.024 \\
PC (40:4) & 2.4 & 0.0000 \\
PC (40:5) & 3.1 & 0.00000 \\
PC (40:6) & 1.8 & 0.0000 \\
PC (40:8) & 2.1 & 0.0000 \\
PC (44:8) & 14 & 0.0000
\end{tabular}

\begin{tabular}{ccl}
\multicolumn{3}{c}{ PE } \\
\hline Metabolite & FC & FDR \\
\hline PE (32:0) & 1.1 & 0.58 \\
PE (34:1) & 1.8 & 0.0000 \\
PE (34:2) & 1.1 & 0.22 \\
PE $36: 1)$ & 1.7 & 0.0000 \\
PE (36:2) & 0.96 & 0.62 \\
PE 36:3) & 1.9 & 0.0000 \\
PE 36:6) & 0.67 & 0.0022 \\
PE 38:1) & 19 & 0.0000 \\
PE 38:3) & 1.9 & 0.0000 \\
PE (38:4) & 1.7 & 0.0000 \\
PE (38:5) & 1.8 & 0.0000 \\
PE (38:6) & 0.83 & 0.11 \\
PE (40:5) & 0.55 & 0.0000 \\
PE (40:7) & 0.96 & 0.78
\end{tabular}

\begin{tabular}{ccl} 
& PS & \\
\hline Metabolite & FC & FDR \\
\hline PS $(34: 2)$ & 0.18 & 0.0000 \\
PS (36:1) & 1.6 & 0.0000 \\
PS (36:4) & 0.99 & 0.90 \\
PS (38:3) & 0.83 & 0.037 \\
PS (38:5) & 1.3 & 0.0044 \\
PS (38:6) & 0.14 & 0.0000 \\
PS (40:5) & 0.70 & 0.013 \\
PS (40:6) & 0.67 & 0.0044 \\
PS (40:7) & 0.59 & 0.0064 \\
& &
\end{tabular}

\begin{tabular}{ccc}
\multicolumn{3}{c}{ Lyso-PC } \\
\hline Metabolite & FC & FDR \\
\hline Lyso-PC (16:0) & 1.5 & 0.020 \\
Lyso-PC (18:0) & 0.31 & 0.0013 \\
Lyso-PC (18:1) & 3.5 & 0.0000 \\
Lyso-PC (18:2) & 2.5 & 0.025 \\
Lyso-PC (22:4) & 9.7 & 0.0000 \\
& &
\end{tabular}

\begin{tabular}{lll}
\multicolumn{4}{c}{ Lyso-PE } \\
\hline Metabolite & FC & FDR \\
\hline Lyso-PE (16:0) & 1.8 & 0.039 \\
Lyso-PE (18:0) & 1.4 & 0.013 \\
Lyso-PE (18:1) & 1.1 & 0.90 \\
Lyso-PE (20:4) & 1.9 & 0.0064
\end{tabular}




\section{Figure 3 - figure supplement 1}

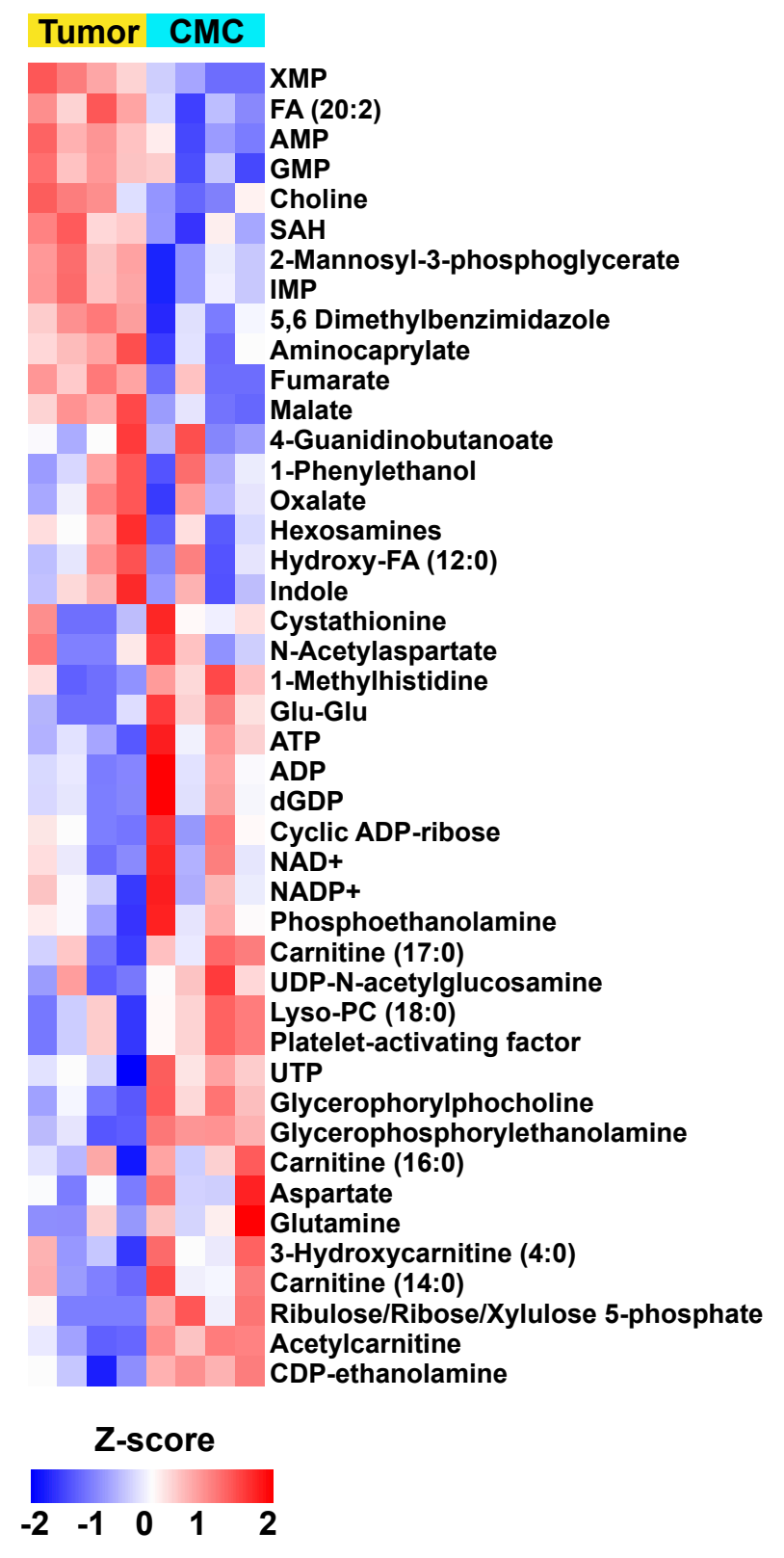




\section{Figure 3 - figure supplement 2}

A Flow cytometry gating strategy for haematopoietic cells
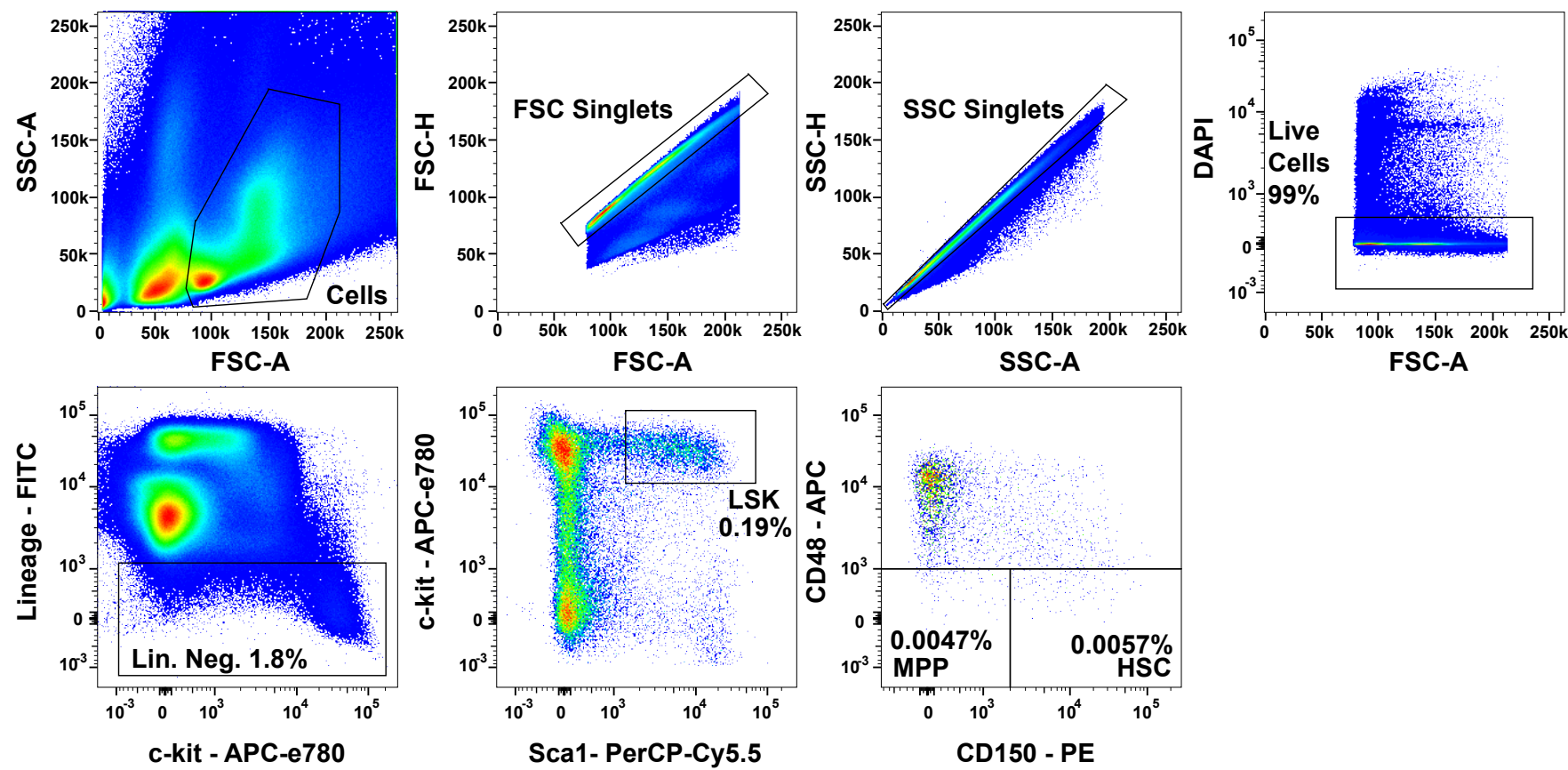

B Flow cytometry gating strategy for human melanoma cells from subcutaneous tumors

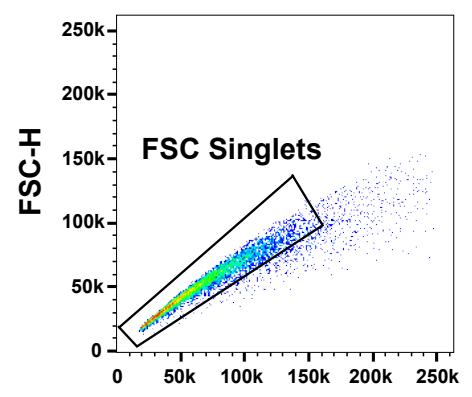

FSC-A
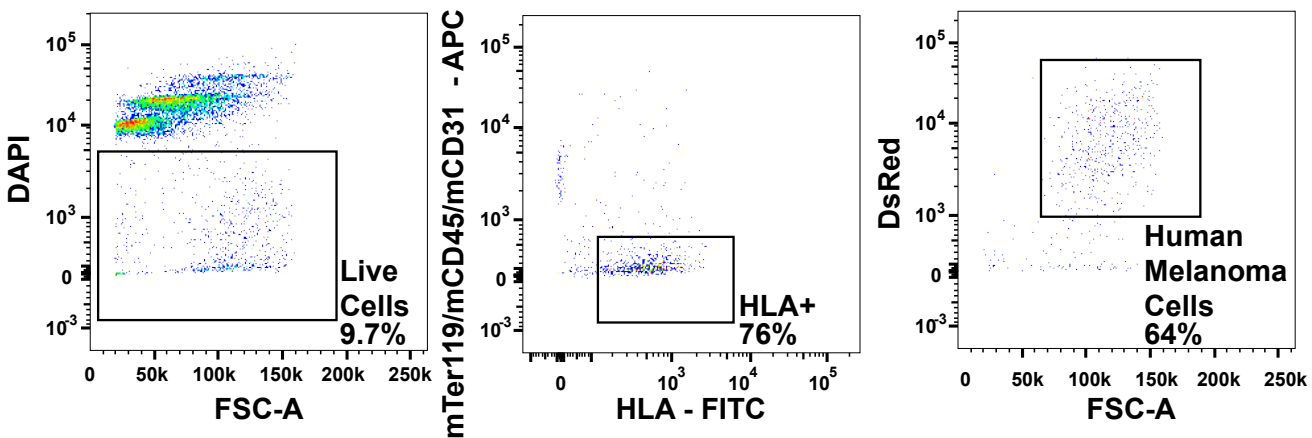

C Flow cytometry gating strategy for circulating human melanoma cells from NSG mouse blood

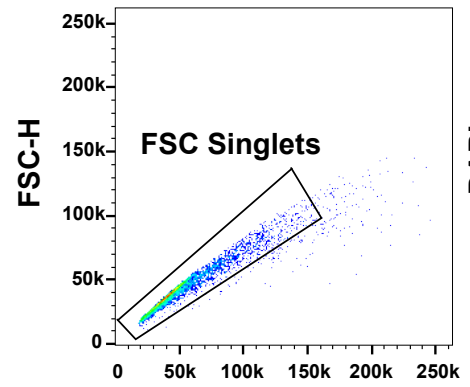

FSC-A

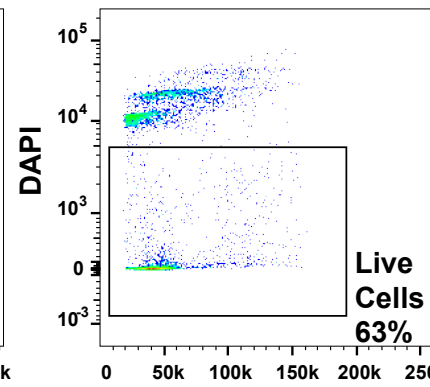

FSC-A

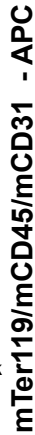

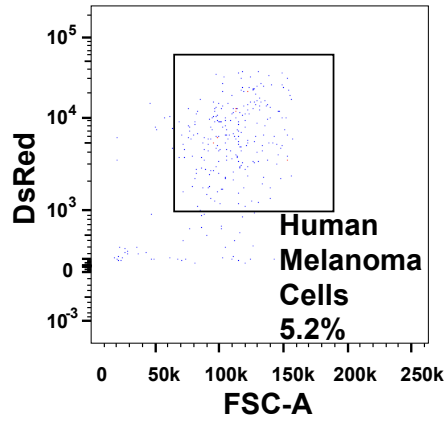

